\title{
Tuning the sensitivity of an optical cavity with slow and fast light
}

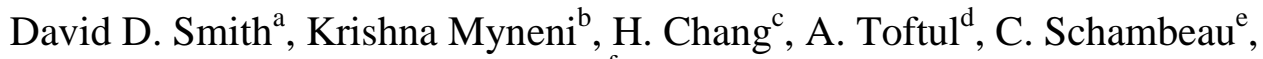 \\ J. A. Odutola ${ }^{f}$ and J. C. Diels ${ }^{\mathrm{g}}$ \\ ${ }^{a}$ Space Systems Department, NASA Marshall Space Flight Center, ES31, Huntsville, AL 35812 \\ ${ }^{b}$ U.S. Army RDECOM, AMSRD-AMR-WS-ST, Redstone Arsenal, AL 35898 \\ ${ }^{c}$ ERC Incorporated, Jacobs ESTS Group, Huntsville, AL 35805 \\ ${ }^{\mathrm{d}}$ Department of Electrical Engineering, University of Nebraska Lincoln, Lincoln, NE 68588 \\ ${ }^{\mathrm{e}}$ Department of Physics, University of Alabama in Huntsville, Huntsville, AL 35899 \\ ${ }^{\mathrm{f}}$ Department of Physics, Chemistry, and Mathematics, Alabama A\&M University, \\ Normal, AL 35762 \\ ${ }^{\mathrm{g}}$ Department of Physics and Astronomy, University of New Mexico, 800 Yale Blvd. NE, \\ Albuquerque, NM 87131
}

\begin{abstract}
We have measured mode pushing by the dispersion of a rubidium vapor in a Fabry-Perot cavity and have shown that the scale factor and sensitivity of a passive cavity can be strongly enhanced by the presence of such an anomalous dispersion medium. The enhancement is the result of the atom-cavity coupling, which provides a positive feedback to the cavity response. The cavity sensitivity can also be controlled and tuned through a pole by a second, optical pumping, beam applied transverse to the cavity. Alternatively, the sensitivity can be controlled by the introduction of a second counter-propagating input beam that interferes with the first beam, coherently increasing the cavity absorptance. We show that the pole in the sensitivity occurs when the sum of the effective group index and an additional cavity delay factor that accounts for mode reshaping goes to zero, and is an example of an exceptional point, commonly associated with coupled nonHermitian Hamiltonian systems. Additionally we show that a normal dispersion feature can decrease the cavity scale factor and can be generated through velocity selective optical pumping.
\end{abstract}

Keywords: Optical Resonators, Laser Gyroscopes, Coherent Optical Effects

\section{INTRODUCTION}

The modification of the response of an optical cavity by an intracavity dispersive medium is of interest for applications such as enhancement of the sensitivity-bandwidth product for interferometric gravity wave detectors, ${ }^{1-3}$ precision measurements of the Lense-Thirring frame-dragging effect, the auto-stabilization of optical cavities, ${ }^{4,5}$ enhanced strain sensing, ${ }^{6}$ and the enhancement of optical gyroscopes. ${ }^{5,7-12}$ In a recent paper the phenomenon of mode pushing in a Fabry-Perot cavity by an intracavity medium with an absorption resonance was demonstrated. ${ }^{9}$ In the region of the absorption resonance, the peaks of the cavity modes are displaced from their empty cavity positions by an amount which depends on the anomalous dispersion associated with the medium's resonance. It was shown that the mode pushing is further enhanced by the variation in the absorption across the finite width of the cavity mode profile which results in a reshaping of the mode. These effects have particular relevance to optical ring cavity devices, in particular to optical gyroscopes and to ring laser gyroscopes. For these sensors the mode pushing phenomenon may be used to enhance both the magnitude of the response to rotation (scale factor) and the signal-to-noise ratio (sensitivity). Fortunately, the essential physics of the mode pushing due to the intracavity dispersive/absorptive medium can be studied simply with a one-dimensional cavity, e.g. a Fabry-Perot cavity with tunable spacing as shown in Fig. 1, and 
does not require assembly of a rotating ring cavity. Neither is a high finesse cavity required. Although for device development of passive resonator gyros, high finesse would ultimately be desirable because the sensitivity scales inversely with mode width, for laboratory studies a low finesse cavity has the advantage that its mode widths are comparable to Doppler-broadened $\mathrm{Rb}$ transition linewidths, making observation and demonstration of the dispersion enhancement considerably more straightforward. In this paper we review our recent results using such a low finesse passive cavity, which demonstrate that the sensitivity, defined as the cavity scale factor divided by the mode width, can be strongly modified the intracavity dispersion, ${ }^{9}$ and examine various methods that may be used for tuning the scale factor. ${ }^{11,12}$

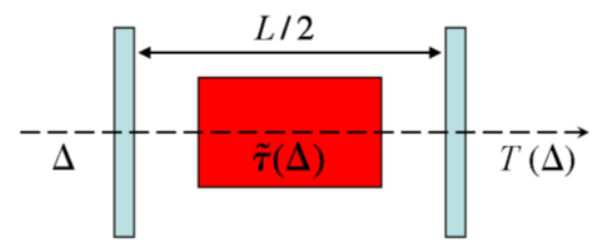

Fig. 1. A Fabry-Perot cavity with an intracavity dispersive medium.

The frequency shift that occurs for a given cavity rotation rate (in the case of a ring cavity) or change in cavity length (for a planar cavity) defines the cavity scale factor. In a previous paper we demonstrated that the scale factor enhancement of a cavity containing an intracavity dispersive medium having a linear complex round trip transmittivity $\tilde{\tau}(\Delta)=\tau(\Delta) \exp [i \Phi(\Delta)]$ where $\Delta=\omega-\omega_{0}$ is the detuning from the medium resonance in angular frequency units, is given by

$$
S\left(\Delta_{p}\right) \equiv \frac{d \Delta_{p}}{d \delta_{q}}=\left[1+T_{d}\left(\Delta_{p}\right)+T_{c a v}\left(\Delta_{p}\right)\right]^{-1}
$$

where $\Delta_{p}=\omega_{p}-\omega_{0}$ and $\delta_{q}=\omega_{q}-\omega_{0}$ are the specific mode detunings with and without the dispersive medium present, respectively. We have written the result in terms of two dimensionless phase times

$$
T_{d}\left(\Delta_{p}\right)=\hat{n}_{g}\left(\Delta_{p}\right)-1=\frac{1}{\tau_{c}} \frac{d \Phi\left(\Delta_{p}\right)}{d \Delta},
$$

and

$$
T_{c a v}\left(\Delta_{p}\right)=\frac{1}{\tau_{c}} \frac{d F\left(\Delta_{p}\right)}{d \Delta},
$$

where $\hat{n}_{g}(\Delta)$ is the effective group index, $\tau_{c}$ is the empty cavity round trip time, and

$$
F(\Delta)=-\sin ^{-1}\left[\frac{1-g(\Delta)^{2}}{2 g(\Delta)} \frac{1}{\hat{n}_{g}(\Delta) \tau_{c}} \frac{d \ln \tau(\Delta)}{d \Delta}\right]
$$

represents an additional cavity feedback phase that must be added to the round trip phase shift $\phi(\Delta)+\Phi(\Delta)$ to obtain the detuning with respect to the pushed mode frequencies, i.e., $\phi(\Delta)+\Phi(\Delta)+F(\Delta)=\tau_{c}\left(\Delta-\Delta_{p}\right)$. The detuning in the absence of the medium is determined simply by the empty cavity round-trip phase shift $\phi(\Delta)=\tau_{c}\left(\Delta-\delta_{q}\right)$. Note that while $\Phi(\Delta)$ depends only on the dispersive medium properties, $F(\Delta)$ depends on the net electric-field gain per round trip $g(\Delta)=\operatorname{ra\tau }(\Delta)$, i.e., it depends on the cavity properties, where $r$ is the round-trip mirror reflectivity, and $a$ accounts for other frequency-independent cavity losses. Hence the factors $F\left(\Delta_{p}\right)$, and $T_{c a v}\left(\Delta_{p}\right)$, account for the variation of absorption across the finite width of the cavity mode, which further increases the mode pushing through a reshaping of the mode profile. When the width of the cavity mode is narrow in comparison with that of the dispersive feature, e.g., in the case of a laser, this mode reshaping can be neglected, and we recover the result in refs [5] and [8], specifically that the scale factor enhancement is inversely proportional to the group index $S^{(L)}\left(\Delta_{p}\right)=1 / \hat{n}_{g}\left(\Delta_{p}\right)$. Given the same level of absorption, larger dispersive feedbacks are therefore obtained for a passive cavity than for a laser. 


\section{SCALE FACTOR POLE, CRITICAL ANOMALOUS DISPERSION AND GAIN, AND EXCEPTIONAL POINTS}

Taking the derivative of Eq. (4) within the approximation of constant group index in the linear regime near resonance we obtain

$$
T_{c a v}\left(\Delta_{p}\right)=\frac{1}{\hat{n}_{g}\left(\Delta_{p}\right) \tau_{c}^{2}}\left[\frac{1-g\left(\Delta_{p}\right)^{2}}{2 g\left(\Delta_{p}\right)}\left(\frac{2 \tau^{\prime}\left(\Delta_{p}\right)^{2}}{\tau\left(\Delta_{p}\right)^{2}}-\frac{\tau^{\prime \prime}\left(\Delta_{p}\right)}{\tau\left(\Delta_{p}\right)}\right)+\frac{\tau^{\prime}\left(\Delta_{p}\right)^{2}}{\tau\left(\Delta_{p}\right)}\right],
$$

where $\tau^{\prime}\left(\Delta_{p}\right)=d \tau\left(\Delta_{p}\right) / d \Delta$ and $\tau^{\prime \prime}\left(\Delta_{p}\right)=d^{2} \tau\left(\Delta_{p}\right) / d \Delta^{2}$. Now we are most interested in the scale factor at resonance, where $\Delta_{p}=0$ and the first derivative of the medium transmission goes to zero, i.e., $\tau^{\prime}(0)=0$. The scale factor on resonance is thus

$$
S_{0}=\frac{1}{\hat{n}_{g}}\left[1-\frac{1-g_{0}{ }^{2}}{2 \hat{n}_{g} \tau_{c}{ }^{2} g_{0}} \frac{\tau_{0}^{\prime \prime}}{\tau_{0}}\right]^{-1} \approx \frac{1}{\hat{n}_{g}}\left[1+\frac{1-g_{0}{ }^{2}}{2 \hat{n}_{g} \tau_{c}{ }^{2} g_{0}} \frac{\tau_{0}^{\prime \prime}}{\tau_{0}}\right],
$$

where we have expanded the result as a geometric series to first order. The series diverges when

$$
\left|\frac{1-g_{0}^{2}}{2 \hat{n}_{g} \tau_{c}^{2} g_{0}} \frac{\tau_{0}^{\prime \prime}}{\tau_{0}}\right| \geq 1
$$

which corresponds to the occurrence of either a pole or a zero in the scale factor depending on whether the term in the absolute value is positive or negative, respectively. Eq. (7) is a transcendental equation that can be rearranged as

$$
g_{c}=\chi+\sqrt{\chi^{2}+1},
$$

where $g_{c}$ is the critical value of the gain corresponding to the scale factor pole and $\chi=-\left(\hat{n}_{g} \tau_{c}\right)^{2} \tau_{0} / \tau_{0}^{\prime \prime}$ is a dispersion parameter that does not depend on the cavity parameters $r$ and $a$. Note that the dispersion parameter is negative for anomalous dispersion so that a solution to Eq. (8) only exists for $g_{c}<1$, i.e., the critical gain always occurs below the lasing threshold. In the limit of weak dispersion, $|\chi| \ll 1$, we obtain $g_{c} \approx 1+\chi$. Note that the critical gain value $g_{c}$ corresponds to a critical value of the group index, or critical anomalous dispersion (CAD), as a result of the KramersKrönig relations. The CAD occurs in general for group indices $0<\hat{n}_{g}<1$, and occurs at $\hat{n}_{g}=0$ only for the case of a laser or when the cavity modes are much narrower than the dispersive feature. For example, for our previously reported experiments, ${ }^{11,12}$ the critical value was $n_{g}^{(c)}=0.67$ for both the $F=1$ to $F^{\prime}$, and $F=2$ to $F$ ' Doppler broadened transitions.

On a final note related to CAD, the scale factor pole is an example of an exceptional point, commonly associated with coupled non-Hermitian Hamiltonian systems, where the complex eigenvalues of the system are fully degenerate. There is, therefore, an accumulated geometric Berry phase as parameters are varied about this exceptional point. For group indices lower than the CAD value (or gain values exceeding $g_{c}$ ), an avoided level crossing is evident by a splitting of the cavity mode. It is straightforward to show that anomalous dispersion corresponds to conservative coupling between the atoms and the cavity mode, whereas normal dispersion corresponds to dissipative coupling.

\section{ENHANCED CAVITY SENSITIVITY}

An enhanced scale factor, by itself, may not result in enhanced measurement sensitivity. For a passive cavity the sensitivity is determined largely by the scale factor $S$, divided by the mode width $W$ (in addition to detector shot noise). Fortunately, whereas the cavity scale factor experiences a pole when $\hat{n}_{g}\left(\Delta_{p}\right)+T_{c a v}\left(\Delta_{p}\right)=0$, the mode width always remains finite. This is because the mode width and scale factor depend differently on the group index as a result of the variation of absorption over the mode width which reshapes the mode as it is swept across the resonance. Our experiments on passive cavities have shown that the sensitivity defined as $\zeta=S / W$ can readily be increased above 
unity by the presence of an intracavity dispersive medium. ${ }^{9,11,12}$ As shown in Fig. 2, the sensitivity in fact has a pole at two distinct values of the net round trip electric field gain $g\left(\Delta_{p}\right):(i)$ at the critical gain $g_{c}$ corresponding to a pole in Eq. (1) owing to the dispersive feedback, and (ii) at the lasing threshold $g\left(\Delta_{p}\right)=1$ as a result of the collapse of the mode width to the Schawlow-Townes linewidth.

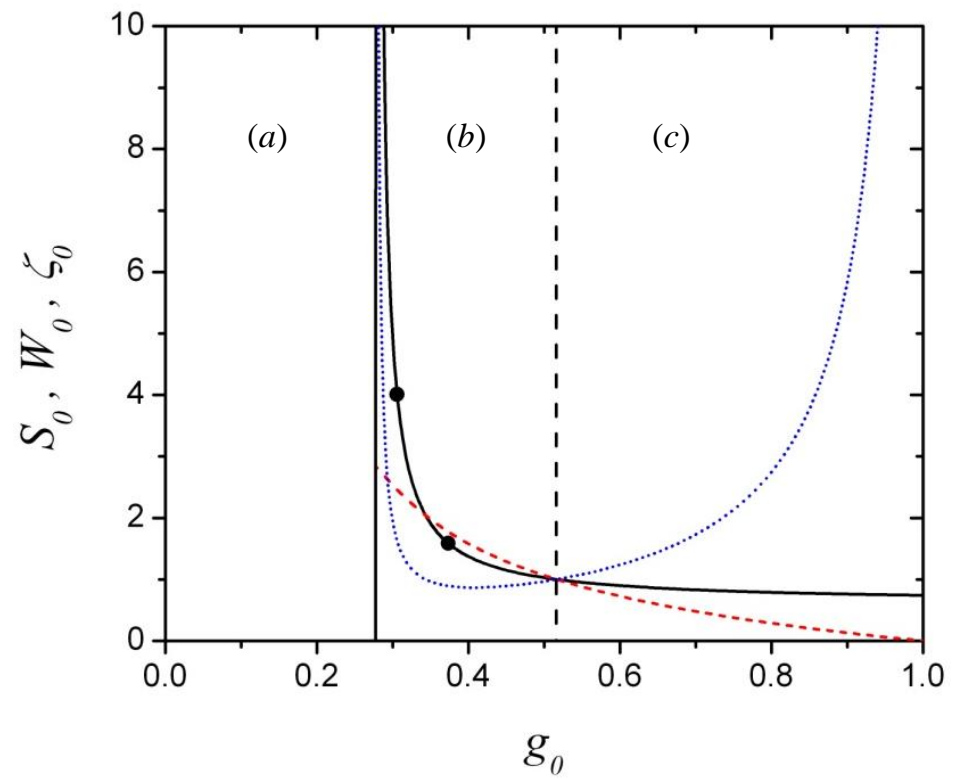

Fig. 2. Scale factor $S_{0}$ (solid curve), normalized mode width $W_{0}$ (dashed curve), and sensitivity $\zeta_{0}$ (dotted curve) versus cavity roundtrip field gain on resonance $g_{0}$ for the $\mathrm{D} 2, F=1$ to $F$ ' Doppler broadened transition, showing regions of (a) mode splitting, (b) mode pushing, and (c) mode pulling. In region (b) the medium is absorbing $\tau_{0}<1$ and the dispersion is anomalous such that $S_{0}>1$. The critical gain occurs at $g_{c}=0.28$ (solid vertical line). In region (a) the anomalous dispersion is so strong that mode splitting occurs. When $g_{0}=r a$ (dashed vertical line) the medium becomes transparent, $\tau_{0}=1$, resulting in the empty cavity case, i.e., $S_{0}=W_{0}=\zeta_{0}=1$. In region (c) the medium is amplifying $\tau_{0}>1$ and normal dispersion results in $S_{0}<1$, but the mode width $W_{0}$ narrows resulting in an increase in sensitivity as the lasing threshold, $g_{0}=1$, is approached. Measurements of $S_{0}$ using a $3 \mathrm{~cm} \mathrm{Rb87}$ vapor cell under different pumping conditions (see next section) are indicated by the two points on the graph.

\section{TUNING BY OPTICAL PUMPING}

Our experiments on unlocked passive cavities have demonstrated that the cavity scale factor can be tuned through its pole by using optical hyperfine pumping to modify the absorption of the intracavity medium. In these experiments a narrow linewidth tunable "probe" diode laser is scanned over the modes of a Fabry-Perot cavity containing an Rb87 cell as described previously. ${ }^{9}$ The detuning between the cavity mode and the atomic resonance, $\delta$, is varied by adjusting the Fabry-Perot cavity length, and spectra are recorded for a variety of detunings to produce a plot of the cavity scale factor as shown in Fig. 3. In Fig. 3a, a scale factor enhancement of $S=4.0$ is evident by the increased slope near the D2, $F=1$ to $F$ ' Doppler broadened resonance, whereas the mode width enhancement, limited by the variation in absorption (as discussed previously), was only $W=2.5$, translating to a sensitivity enhancement of $\zeta=S / W=1.6$ for this case. A second tunable diode laser, locked to a pumping transition (e.g., $F=1$ to $F^{\prime}=2$ ), provides a pump beam of adjustable intensity that is focused via a cylindrical lens into the intracavity vapor cell, in a direction orthogonal to the probe beam. The orthogonality of the pump and probe beams means that all velocity groups sampled by the probe beam are pumped, i.e., the pumping is not velocity-selective along the probe propagation axis. As a consequence of this arrangement, no additional resonances occur in the probe spectrum (in contrast with the velocity-selective collinear pumping scheme 
outlined in section 5). Hence the pump beam shifts uniformly the optical transmission of the Rb87 vapor, which in turn modifies the cavity scale factor. As shown in Fig. 3b, the scale factor can either increase or decrease depending on the choice of pumping transition, and can be tuned continuously simply by changing the pump intensity. This continuous tuning capability could be used to adaptively change the sensitivity of an optical gyroscope, increasing it for low rotation rates and decreasing it for large rotation rates, increasing its effective dynamic range. Furthermore, the time scale for changing the sensitivity with optical pumping can be on the order of one microsecond or less, with moderate pump intensities. This allows for rapid tuning of the cavity optical response, which can be important for gyroscopic applications in highly dynamic environments.
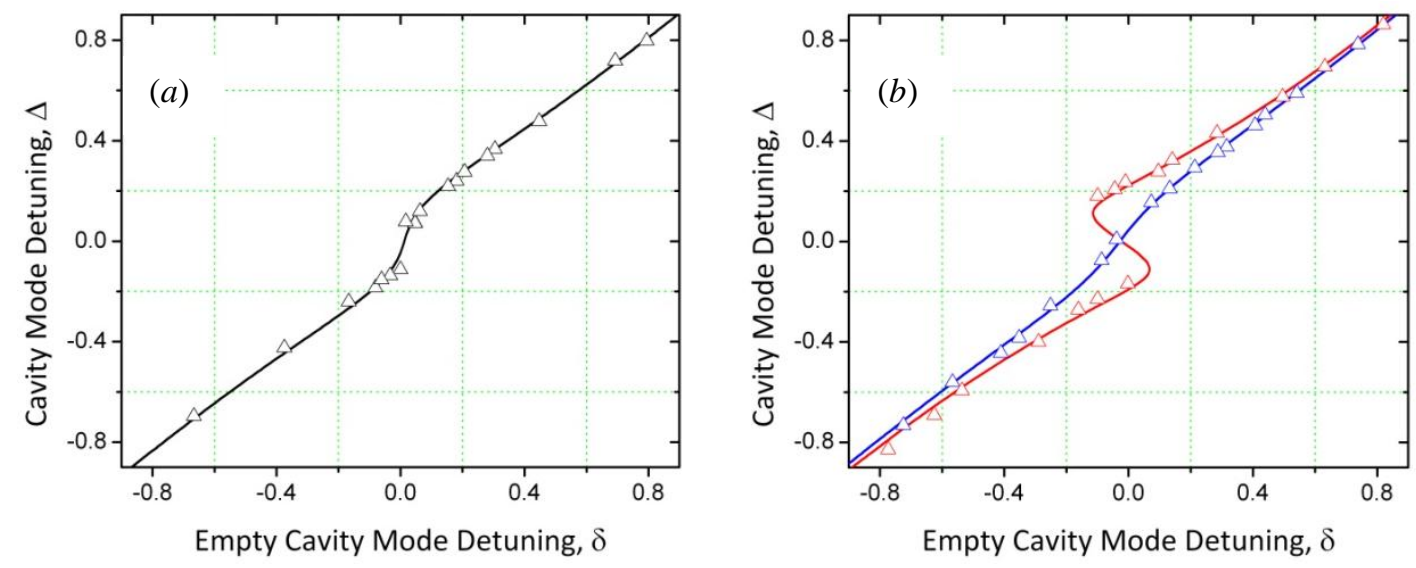

Fig. 3. Cavity scale factor enhancement, i.e., dispersive vs. empty cavity mode detuning for a $3 \mathrm{~cm}{ }^{87} \mathrm{Rb}$ cell in a $15 \mathrm{~cm}$ Fabry-Perot cavity (a) without pump beam and (b) with pump beam locked to the $F=1$ to $F^{\prime}=2$ (blue curve) and $F=2$ to $F^{\prime}=2$ (red curve) transitions. In (a) near the ${ }^{87} \mathrm{Rb} \mathrm{D} 2, F=1$ to $F^{\prime}$ Doppler broadened resonance, the scale factor is increased by anomalous dispersion. In (b) the scale factor is strongly affected by the presence of the pump beam and can be either decreased (blue curve) or increased until it passes through a pole resulting in mode splitting (red curve).

Data were also obtained with cavity modes tuned across the D2, $F=2$ to $F^{\prime}$ Doppler broadened resonance. ${ }^{11,12}$ In this case, when the pump was locked to the $F=2$ to $F^{\prime}=2$ transition, we obtained a scale factor of $S=14.8$ and width increase of $W=2.8$, resulting in a sensitivity enhancement of $\zeta=S / W=5.2$.

\section{TUNING BY COHERENT CONTROL OF ABSORPTION}

An alternative method to the use of a pump beam for tuning the cavity sensitivity is to use a second input "probe" beam that coherently interferes with the first probe beam. Recent experiments by us and others have shown that the absorptance of a cavity can be enhanced and even become unity (a perfect absorber), in particular when a second beam of the correct amplitude and phase is incident upon the cavity. ${ }^{13-15}$ At this point, even though the intracavity material itself may not be a strong absorber, the cavity coherently absorbs all the incident radiation falling upon it in a process that is the time reverse of a laser (where a cavity emits coherent radiation). This coherent perfect absorption (CPA) essentially results from two beam interference: the transmittance of the first beam and the reflectance of the second beam destructively interfere. More precisely, when there is only one beam incident on the cavity, the peaks and widths of the cavity modes are determined simply by the complex eigenvalues of the cavity. With two incident beams, on the other hand, the cavity transmission is determined not only by the cavity eigenvalues, but also by the interference of the two inputs. Therefore, the cavity absorptance, and consequently, the scale factor are controllable simply by varying the intensity or phase of the second beam, as shown in Fig. 4. This method has the advantage over the hyperfine pumping method in that it is completely linear and occurs irrespective of the choice of intracavity medium. 

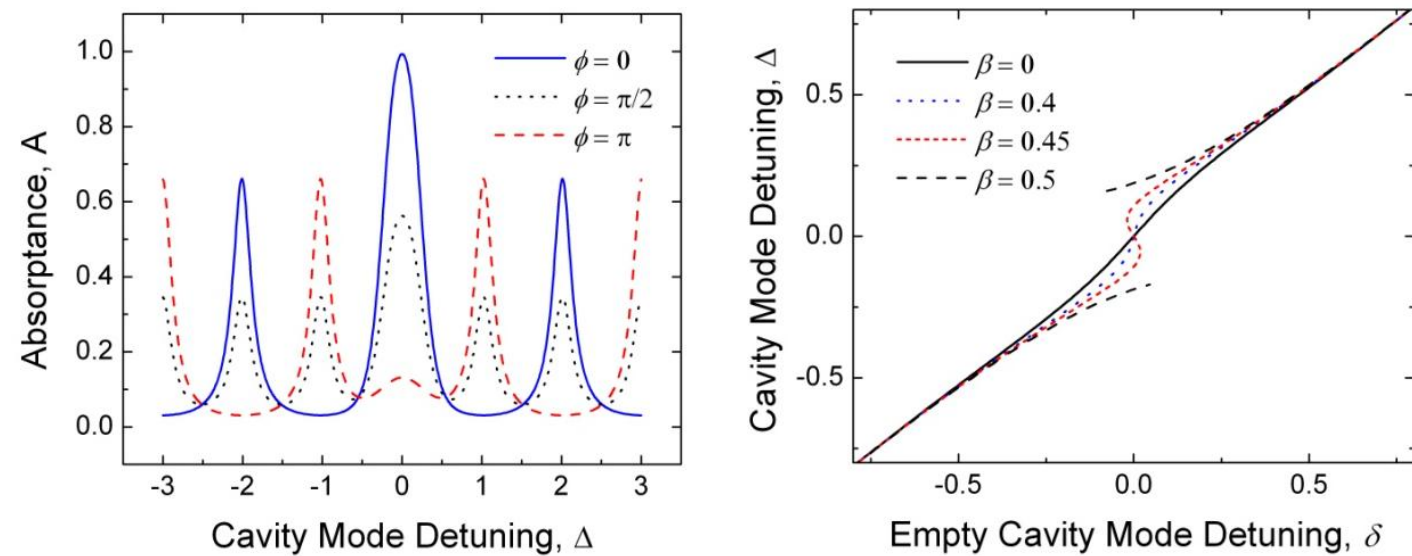

Fig. 4. Absorptance (left) and scale factor (right) for a symmetric cavity with two inputs containing a dispersive medium. Left: Absorptance at $\delta=0$ for equal input amplitudes, $\beta=1$, and various values of the relative phase $\varphi=0, \varphi= \pm \pi / 2$, and $\varphi= \pm \pi$. At $\varphi=0 \quad \varphi= \pm \pi$ the absorptance is enhanced (suppressed) at even detunings and suppressed (enhanced) at odd detunings. All detunings are in FSR units. Hence, the absorption can be toggled on or off at any given cavity mode simply by changing the phase (or amplitude) of the second beam. Note also that CPA $(\mathrm{A}=1)$ is observed on resonance when $\varphi=0$. Right: Scale factor for a fixed phase, $\varphi=0$, and various values of the relative amplitude, $\beta=0$ (single input), $\beta=0.4, \beta=0.45$, and $\beta=0.5$. The scale factor is tuned by varying the intensity of the second input.

\section{DECREASING THE CAVITY SCALE FACTOR}

For many applications it is instead important to decrease the scale factor of a cavity, thereby stabilizing it against unintended motion of the cavity mirrors over some finite bandwidth. Since anomalous dispersion can be used to increase scale factor via mode pushing, the question that arises is whether decreased scale factors can be achieved by the mode pulling associated with the introduction of a normal dispersion medium. In Fig. 5a, we introduce a hypothetical normal dispersion feature modeled as a single Lorentzian function on top of our Doppler broadened transition, and calculate the transmission of the cavity. The effect of the normal dispersion is clearly observed. The mode narrows as it is pulled towards and held by the narrow normal dispersion feature, until the detuning becomes so large that it is finally pushed away by the broader anomalous dispersion feature. This results in a flattening in the scale factor plot as shown in Fig. 5b in a manner that is analogous to the formation of a dead-band in a passive gyro.
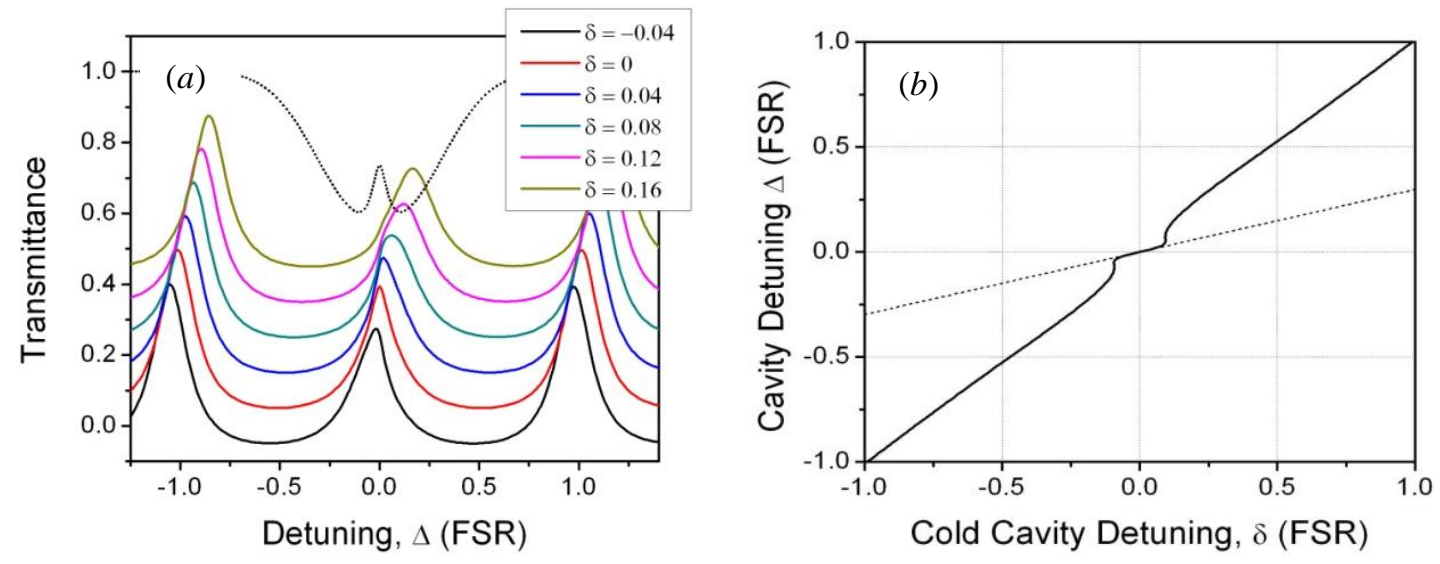

Fig. 5. (a) Cavity transmittance as a cavity mode is swept through an atomic resonance having a narrow normal dispersion feature. Near the resonance the mode is pulled and trapped by the normal dispersion, then abruptly 
released and pushed away by the broader anomalous dispersion feature. (b) Scale factor for a cavity containing a medium having a normal dispersion feature. The scale factor is significantly reduced near the resonance.

Such a normal dispersion feature can be readily produced in the Rb87 absorption spectrum using the technique of velocity selective optical hyperfine pumping. To generate the normal dispersion feature the pump beam is locked to a hyperfine transition that pumps atoms out of the given probe transition (Fig. 6b), resulting in a decrease in the absorption of the probe and the appearance of a normal dispersive feature in the Rb87 absorption spectrum. The pump beam used to produce the normal dispersion feature enters the Rb87 cell collinear to the probe beam in order to affect atoms over a narrow range of velocities. We have observed modest decreases in cavity scale factor using this method.
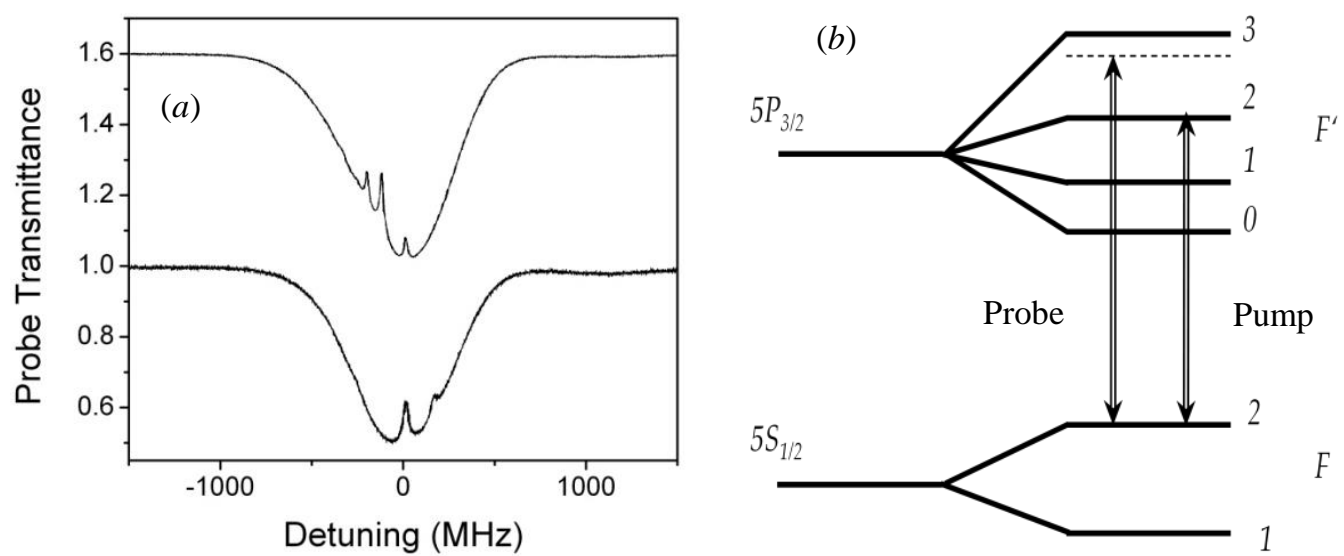

Fig. 6. (a) Bottom: $F=2$ probe transmission spectrum. The pump beam is locked to the ${ }^{87} \mathrm{Rb} F=2$ to $F^{\prime}=2$ hyperfine resonance, generating a strong normal dispersion feature at the $F=2$ to $F^{\prime}=3$ transition in the probe spectrum. The second smaller feature results from the transfer of a second group of atoms whose velocity is such that the pump is resonant with the $F=2$ to $F^{\prime}=1$ transition. Top: the saturated absorption spectrum is also provided for reference. (b) ${ }^{87} \mathrm{Rb} \mathrm{D} 2$ transition energy level diagram showing pump and probe transitions.

\section{CONCLUSION}

In this paper we have reviewed experiments that demonstrate how the sensitivity of the modes of an optical cavity can be increased by anomalous dispersion and tuned by the application of a second pumping or interfering beam. We have also introduced a method for decreasing the cavity scale factor using velocity selective optical pumping. The observed behaviors are not unique to the coupling of atoms and photons in a cavity, but are fundamental to systems of coupled oscillators and are therefore ubiquitous in nature.

\section{ACKNOWLEDGMENTS}

This work was sponsored by ILIR at the AMRDEC. A. Toftul acknowledges funding by the Nebraska Space Grant and NASA MSFC Education Programs Office. J. A. Odutola was supported by the NASA Administrator Fellows Program. 


\section{REFERENCES}

[1] Salit, M., Pati, G. S., Salit, K. and Shahriar, M. S., "Fast-light for astrophysics: super-sensitive gyroscopes and gravitational wave detectors," J. Mod. Opt. 54, 2425-2440 (2007).

[2] Shahriar, M. S. and Salit, M. "Application of fast light in gravitational wave detection with interferometers and resonators," J. Mod. Opt. 55, Nos. 19-20, 10-20, 3133-3147 (2008).

[3] Salit, M. and Shahriar M. S., "Enhancement of sensitivity-bandwidth product of interferometric gravitational wave detectors using white light cavities," J. Opt. 12, 104014 (2010).

[4] Lukin, M. D., Fleischhauer, M., Scully, M. O. and Velichansky, V. L., "Intracavity electromagnetically induced transparency," Opt. Lett. 23, 295-297 (1998).

[5] Smith, D. D., Chang, H., Arissian, L. and Diels, J. C., "Dispersion enhanced laser gyroscope," Phys. Rev. A 78, 053824 (2008).

[6] Pati, G. S., Salit, M., Salit, K and Shahriar, M. S., "Demonstration of displacement-measurement-sensitivity proportional to inverse group index of intra-cavity medium in a ring resonator," Opt. Comm. 281, 4931 (2008).

[7] Leonhardt, U. and Piwnitski, P., "Ultrahigh sensitivity of slow-light gyroscope," Phys. Rev. A 62, 055801 (2000).

[8] Shahriar, M. S., Pati, G. S., Tripathi, R., Gopal, V., Messall, M. and Salit, K., "Ultrahigh enhancement in absolute and relative rotation sensing using fast and slow light," Phys. Rev. A 75, 053807 (2007).

[9] Smith, D. D., Myneni, K., Odutola, J. A. and Diels, J. C., "Enhanced sensitivity of a passive optical cavity by an intracavity dispersive medium," Phys. Rev. A 80, 011809(R) (2009).

[10] Yum, H. N., Salit, M., Yablon, J., Salit, K., Wang Y. and Shahriar, M. S., "Superluminal ring laser for hypersensitive sensing, “Opt. Expr. 18, 17658-17665 (2010).

[11] Myneni, K., Smith, D. D., Odutola, J. A. and Schambeau, C. A., "Tuning the response of an optical cavity using laser pumping of an intracavity atomic vapor," Proc. 27th Army Science Conference, Orlando, FL (2010).

[12] Myneni, K., Smith, D. D., Odutola, J. A. and Schambeau, C. A., "Tuning the scale factor and sensitivity of a passive cavity with optical pumping," Sub. to Phys. Rev. A (2011).

[13] Chong, Y. D., Ge, L., Cao, H. and Stone, A. D., "Coherent perfect absorbers: time-reversed lasers" Phys. Rev. Lett. 105, 053901 (2010).

[14] Wan, W., Chong, Y. D., Ge, L., Noh, H., Stone, A. D. and Cao, H., "Time-reversed lasing and interferometric control of absorption" Science 331, 889 (2011).

[15] Smith, D. D., Myneni, K., Chang, H. and Odutola, J. A., "Coherent enhanced absorption in an Intracavity Atomic Medium," 42nd Annual Meeting of the APS Division of Atomic, Molecular and Optical Physics, Atlanta, GA, Bulletin of the American Physical Society, Vol. 56, Number 5, June 13-17 (2011). 
Tuning the sensitivity of an optical cavity with slow and fast light

David D. Smith

Optics, Imaging, and Sensors Branch NASA Marshall Space Flight Center 


\section{Outline}

> Scale factor of a dispersive optical cavity

$>$ Critical gain/anomalous dispersion

$>$ Tuning by non-velocity-selective optical pumping

$>$ Scale factor for two coupled oscillators

$>$ Decreasing the scale factor by normal dispersion via velocity-selective optical pumping

$>$ Tuning by coherent control of absorption

$>$ Closing the loop 
Mode Pushing / Pulling

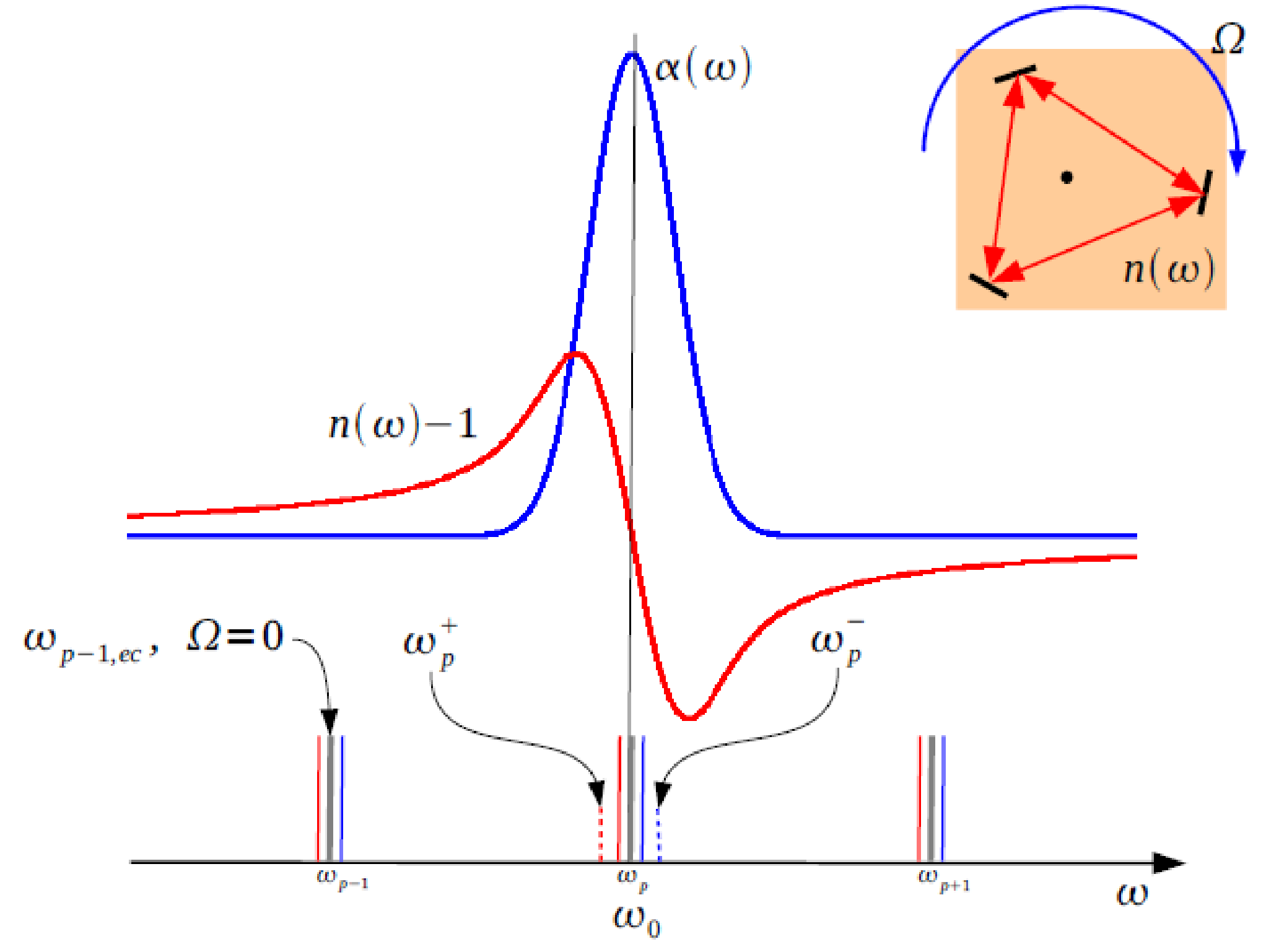


- Question: How does a dispersive material change the frequency and width of the modes of a passive optical cavity?

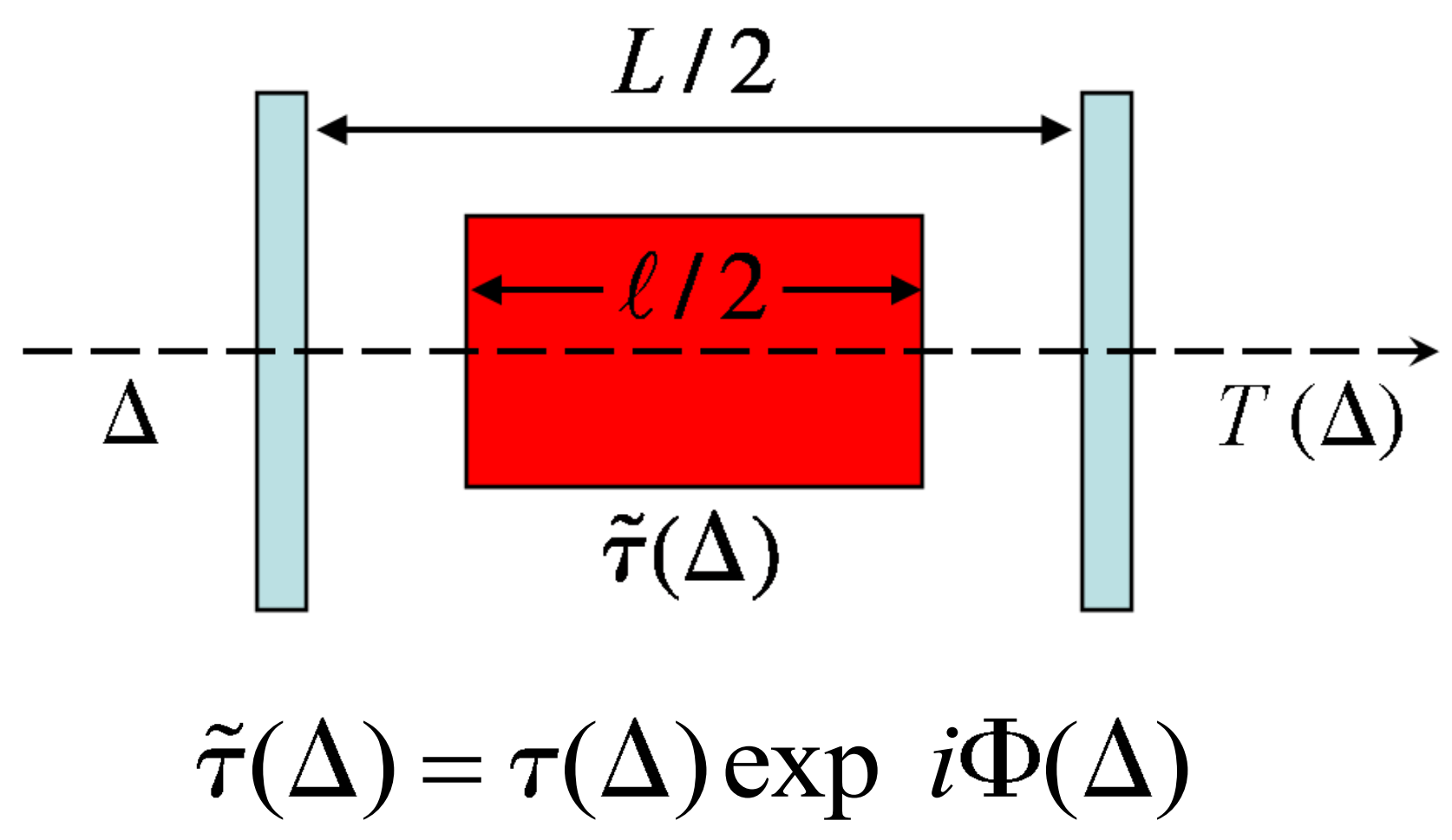

D. D. Smith, K. Myneni, J. Odutola, J. C. Diels, PRA (2009). 


\section{Cavity Transmission}

$$
T\left(\Delta, \delta_{q}\right)=\frac{(1-r)^{2} / r}{1-g(\Delta)^{2} / g(\Delta)+4 \sin ^{2}\left[\frac{\phi(\Delta)+\Phi(\Delta)}{2}\right]}
$$

Cavity

Transmittance

where $g(\Delta)=\operatorname{ra\tau }(\Delta)$ is the net round-trip gain.

Maxima occur at $\Delta_{p}=\delta_{q}-\frac{\left[\Phi\left(\Delta_{p}\right)+F\left(\Delta_{p}\right)+2 \pi p^{\prime}\right]}{\tau_{c}}$ where

$$
F\left(\Delta_{p}\right)=-\sin ^{-1}\left[\frac{1-g\left(\Delta_{p}\right)^{2}}{2 g\left(\Delta_{p}\right)} \frac{1}{\tau_{c} \hat{n}_{g}\left(\Delta_{p}\right)} \frac{d \ln \tau\left(\Delta_{p}\right)}{d \Delta}\right]
$$

Cavity Feedback

$$
\begin{aligned}
& \tau_{c}\left(\Delta-\Delta_{p}\right)=\phi+\Phi(\Delta)+F(\Delta) \quad \text { Detuning } w / \text { Dispersion } \\
& \tau_{c}\left(\Delta-\delta_{q}\right)=\phi(\Delta) \quad \text { Detuning w/o Dispersion }
\end{aligned}
$$




\section{Cavity Scale Factor}

$$
S\left(\Delta_{p}\right) \equiv \frac{d \Delta_{p}}{d \delta}=\left[1+T_{d}\left(\Delta_{p}\right)+T_{c a v}\left(\Delta_{p}\right)\right]^{-1}
$$

Cavity Scale Factor

where the dimensionless phase times are

$$
T_{d}\left(\Delta_{p}\right)=\hat{n}_{g}\left(\Delta_{p}\right)-1=\frac{1}{\tau_{c}} \frac{d \Phi\left(\Delta_{p}\right)}{d \Delta} \quad T_{c a v}\left(\Delta_{p}\right)=\frac{1}{\tau_{c}} \frac{d F\left(\Delta_{p}\right)}{d \Delta_{p}}
$$

For a laser: $g\left(\Delta_{p}\right)=1$ such that $F\left(\Delta_{p}\right)=0$ and

$$
S_{L}\left(\Delta_{p}\right)=\frac{1}{\hat{n}_{g}\left(\Delta_{p}\right)} \quad \begin{gathered}
\text { Cavity Scale } \\
\text { Factor for Laser }
\end{gathered}
$$

M. S. Shahriar, et al., PRA 75, 053807 (2007); D. D. Smith et al. PRA 78, 053824 (2008). 


\section{Experiment}

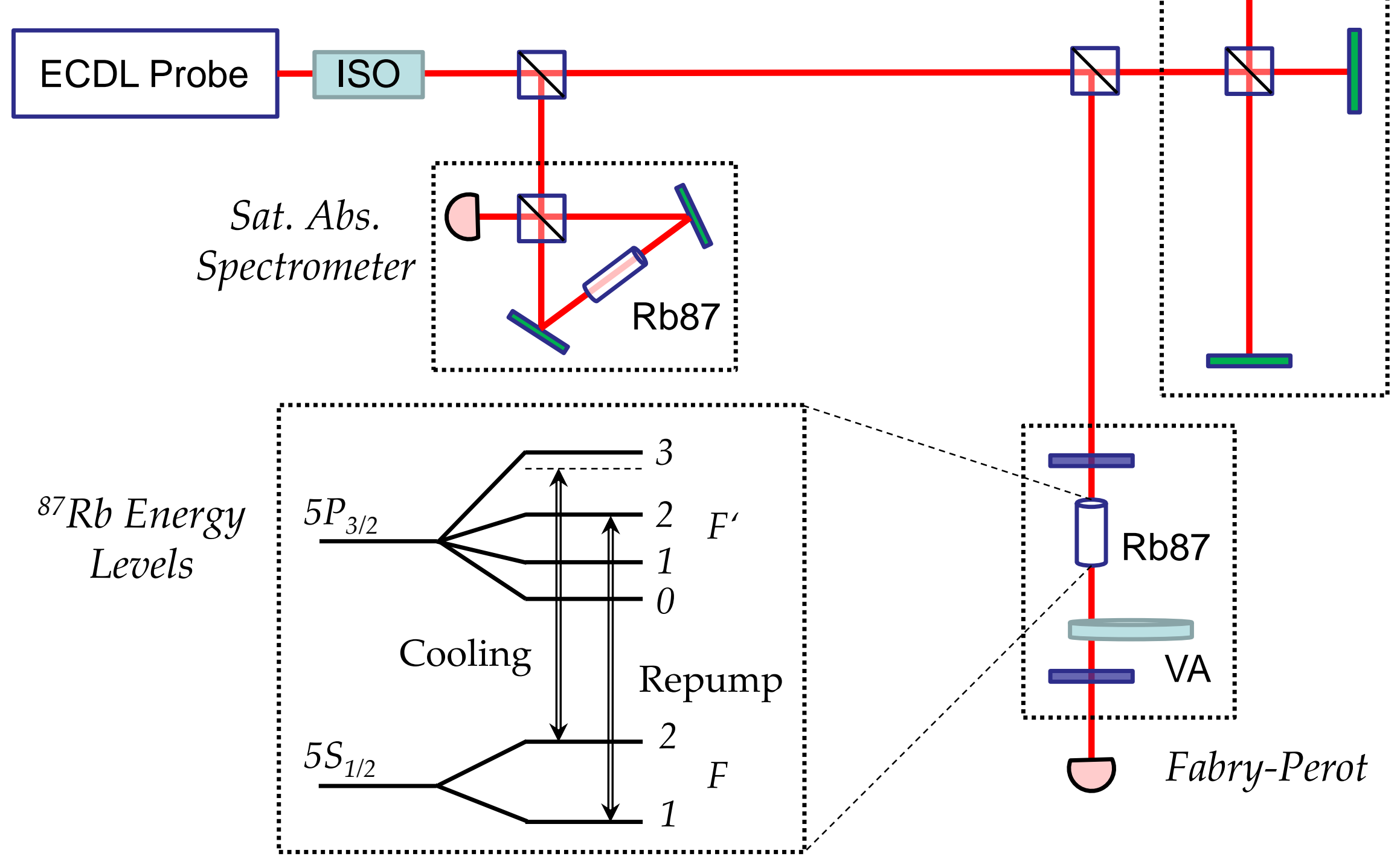




\section{Mode Pushing and Splitting}

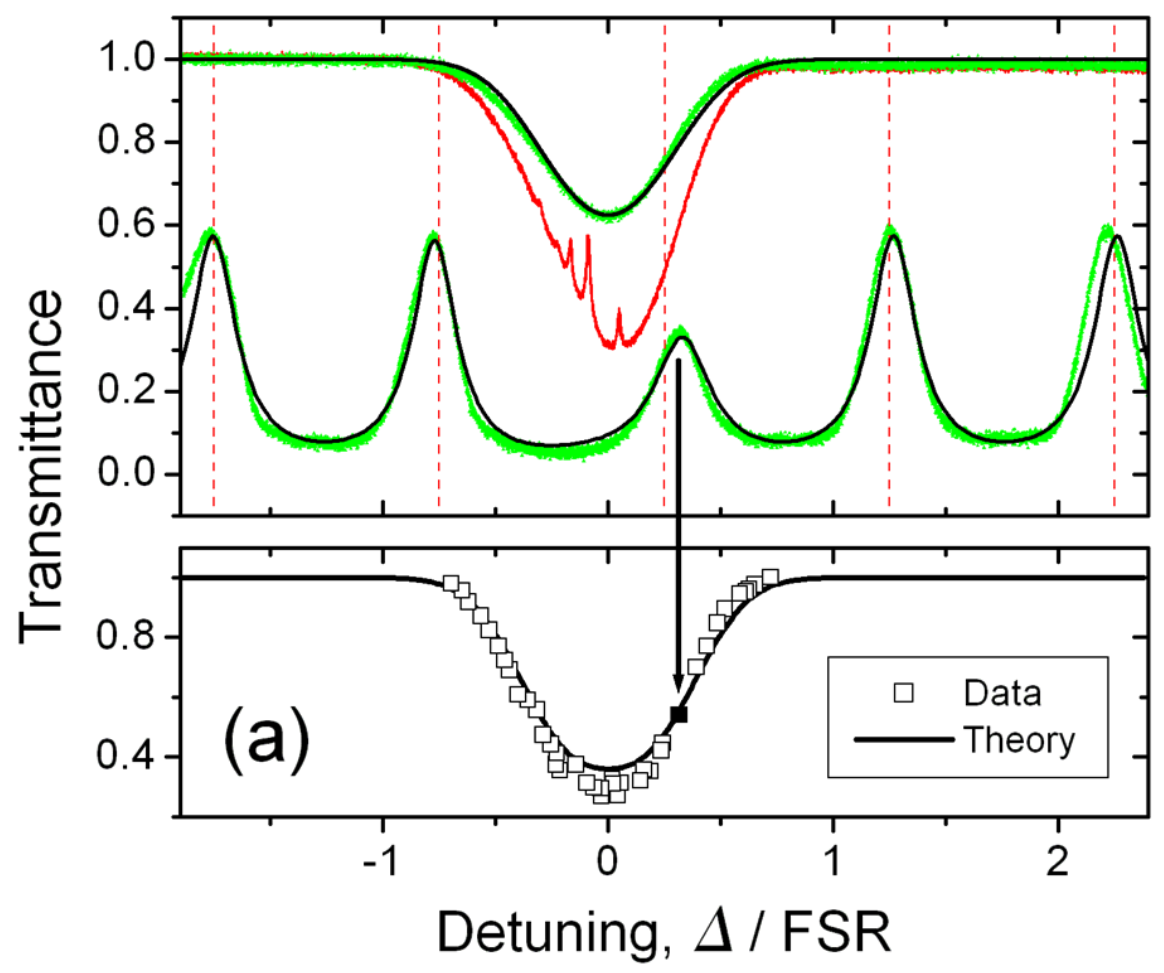

$3 \mathrm{~cm}$ cell

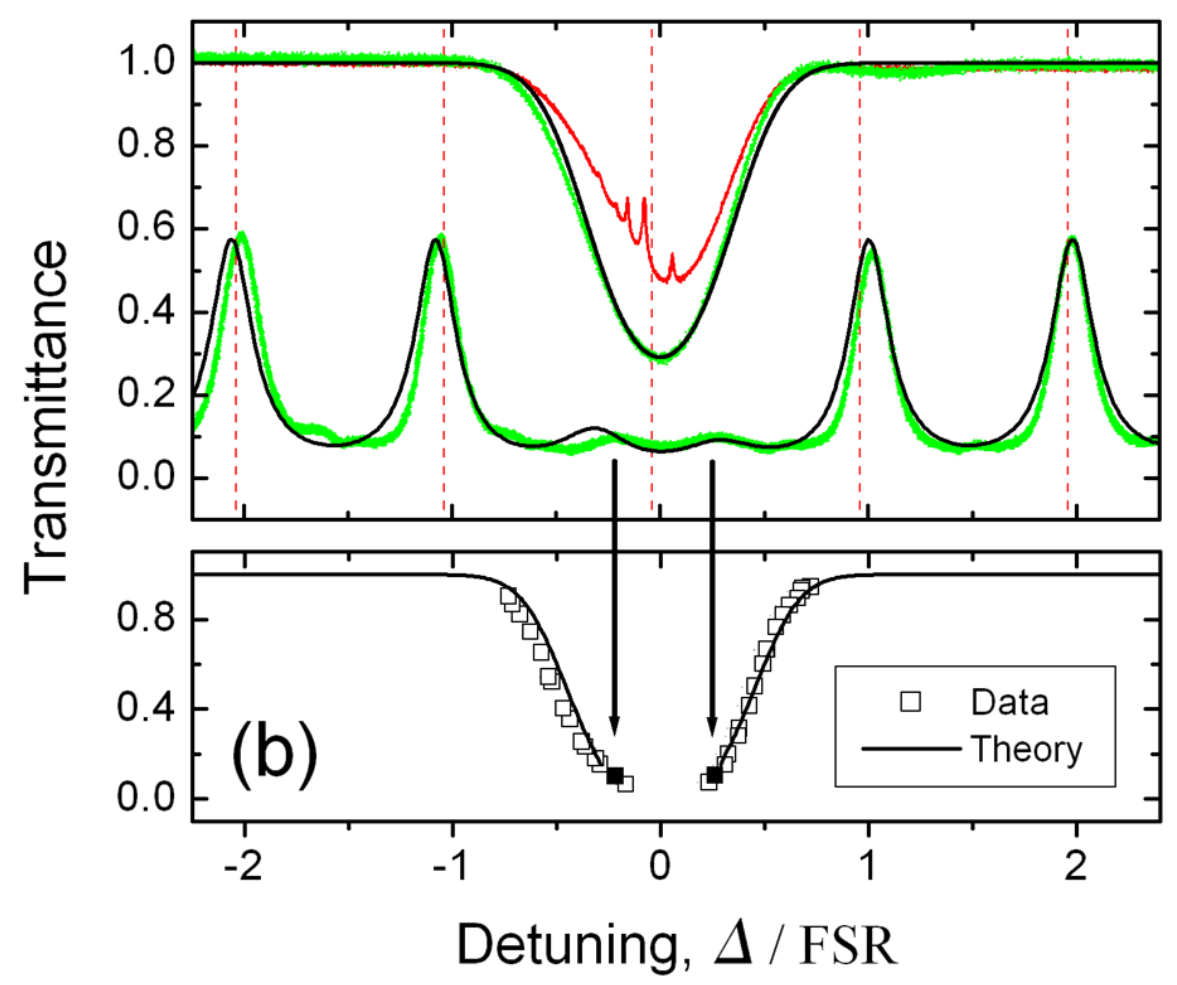

$8 \mathrm{~cm}$ cell

D. D. Smith, K. Myneni, J. Odutola, J. C. Diels, PRA (2009). 


\section{Scale Factor: Enhancement}

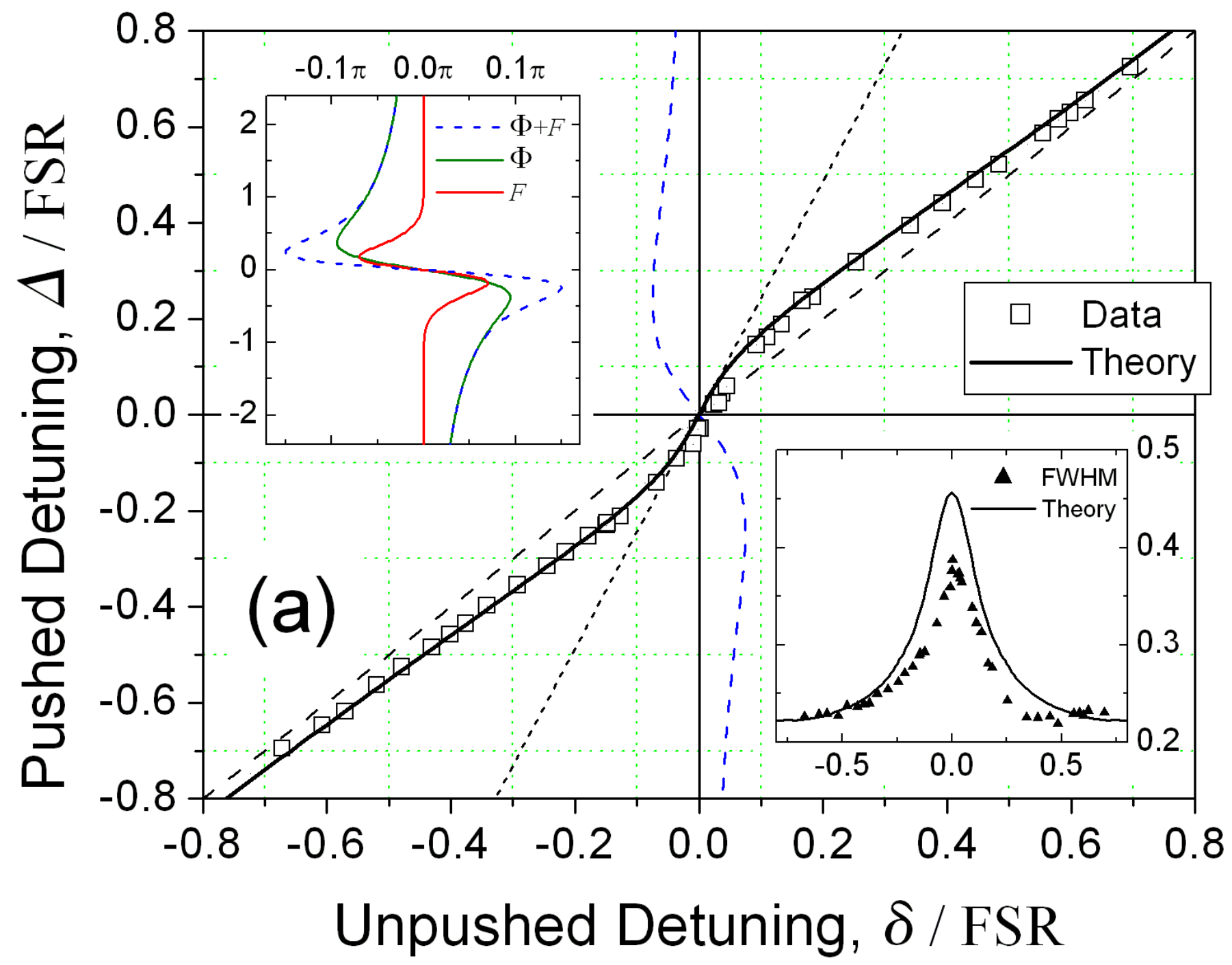

$3 \mathrm{~cm}$ cell

$\hat{n}_{g}(0)=0.77$ 


\section{Scale Factor: Mode Splitting}

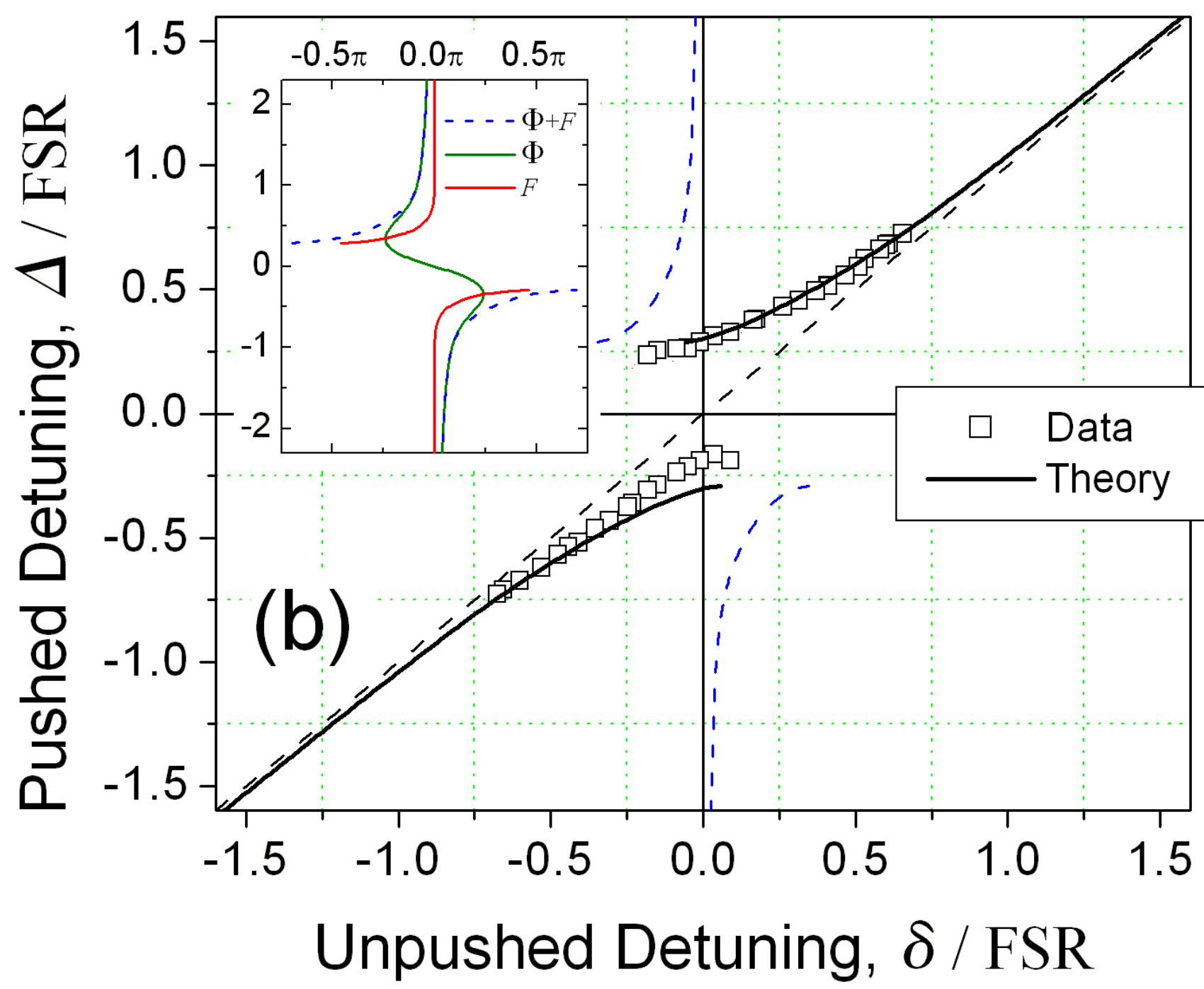

$8 \mathrm{~cm}$ cell

$\hat{n}_{g}(0)=0.32$ 


\section{Critical Gain}

$S_{0}=\frac{1}{\hat{n}_{g}}\left[1-\frac{1-g_{0}{ }^{2}}{2 \hat{n}_{g} \tau_{c}{ }^{2} g_{0}} \frac{\tau_{0}^{\prime \prime}}{\tau_{0}}\right]^{-1} \approx \frac{1}{\hat{n}_{g}}\left[1+\frac{1-g_{0}{ }^{2}}{2 \hat{n}_{g} \tau_{c}{ }^{2} g_{0}} \frac{\tau_{0}^{\prime \prime}}{\tau_{0}}\right] \begin{aligned} & \text { Cavity } \\ & \begin{array}{l}\text { Scale } \\ \text { Factor }\end{array}\end{aligned}$

the series diverges for:

$$
\left|\frac{1-g_{0}^{2}}{2 \hat{n}_{g} \tau_{c}^{2} g_{0}} \frac{\tau_{0}^{\prime \prime}}{\tau_{0}}\right| \geq 1
$$

positive $\rightarrow$ pole

negative $\rightarrow$ zero
Critical gain condition

> Solution to positive transcendental equation yields the critical gain or critical anomalous dispersion that results in a scale factor pole (also an exceptional point) :

$$
g_{c}=0.28 \quad n_{g}^{(c)}=0.67
$$




\section{Cavity Sensitivity}

Round-Trip electric field gain, $g(0)$

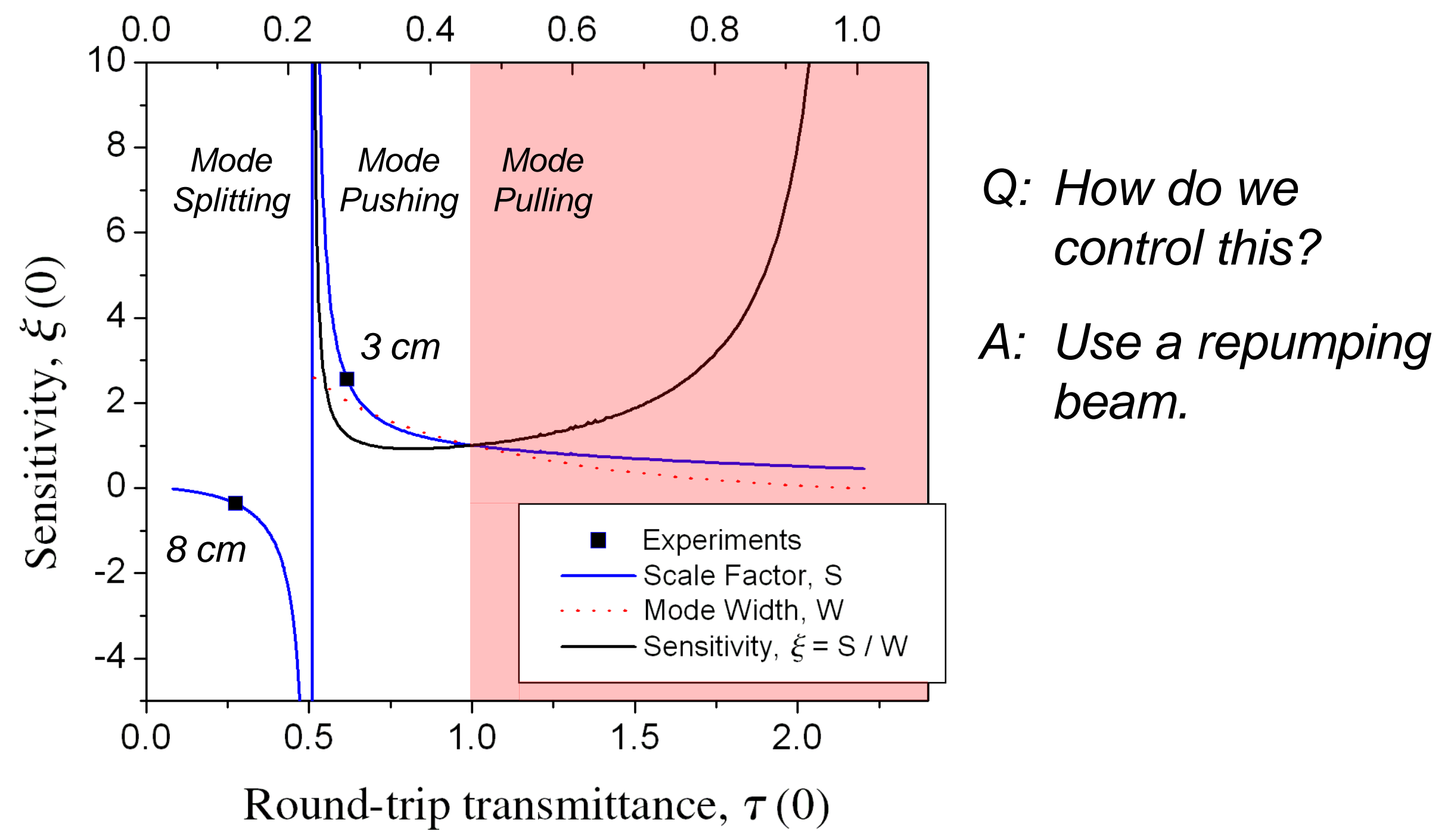




\section{Experiment}

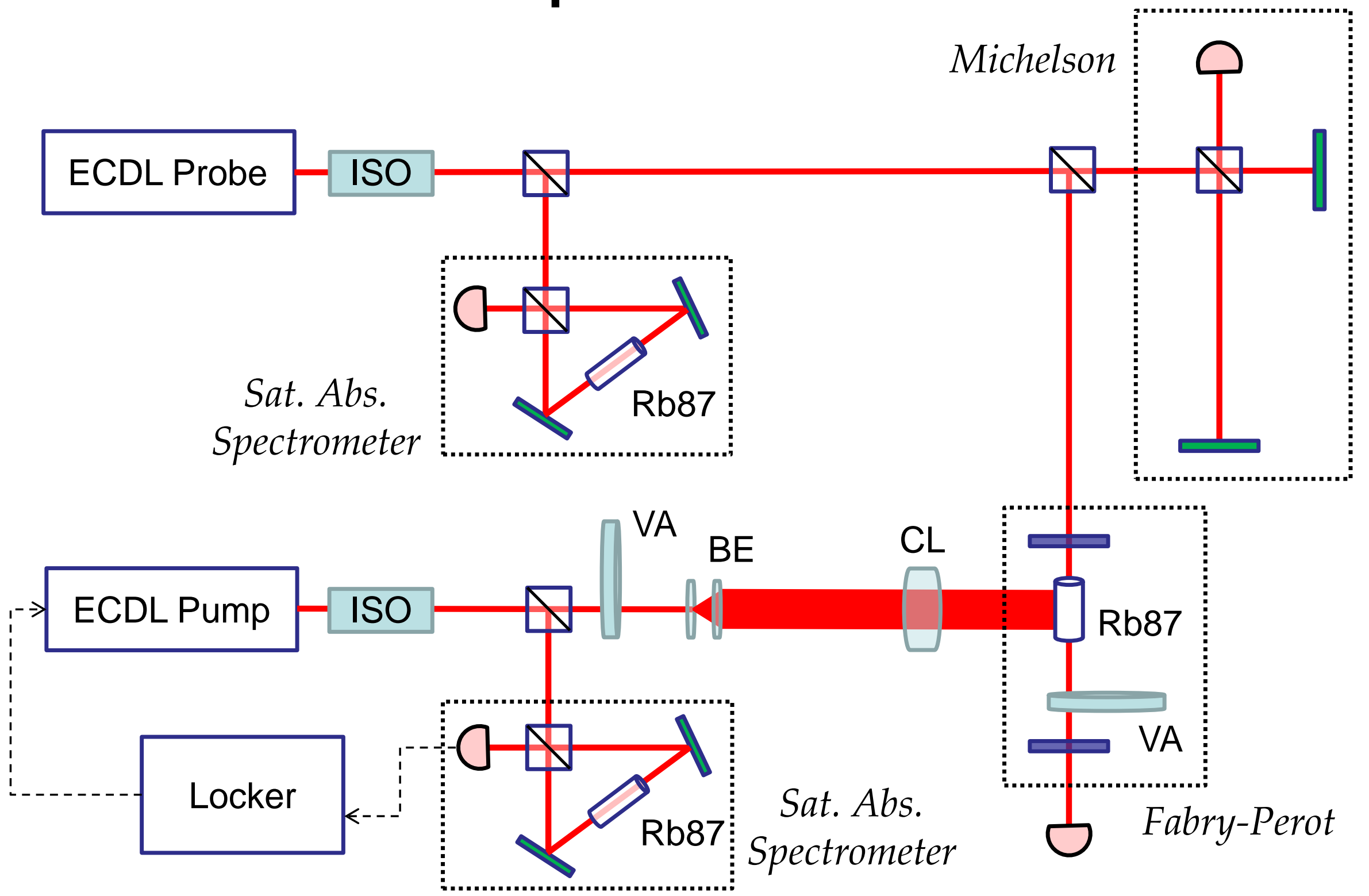




\section{Rb87 Transmission Spectra}
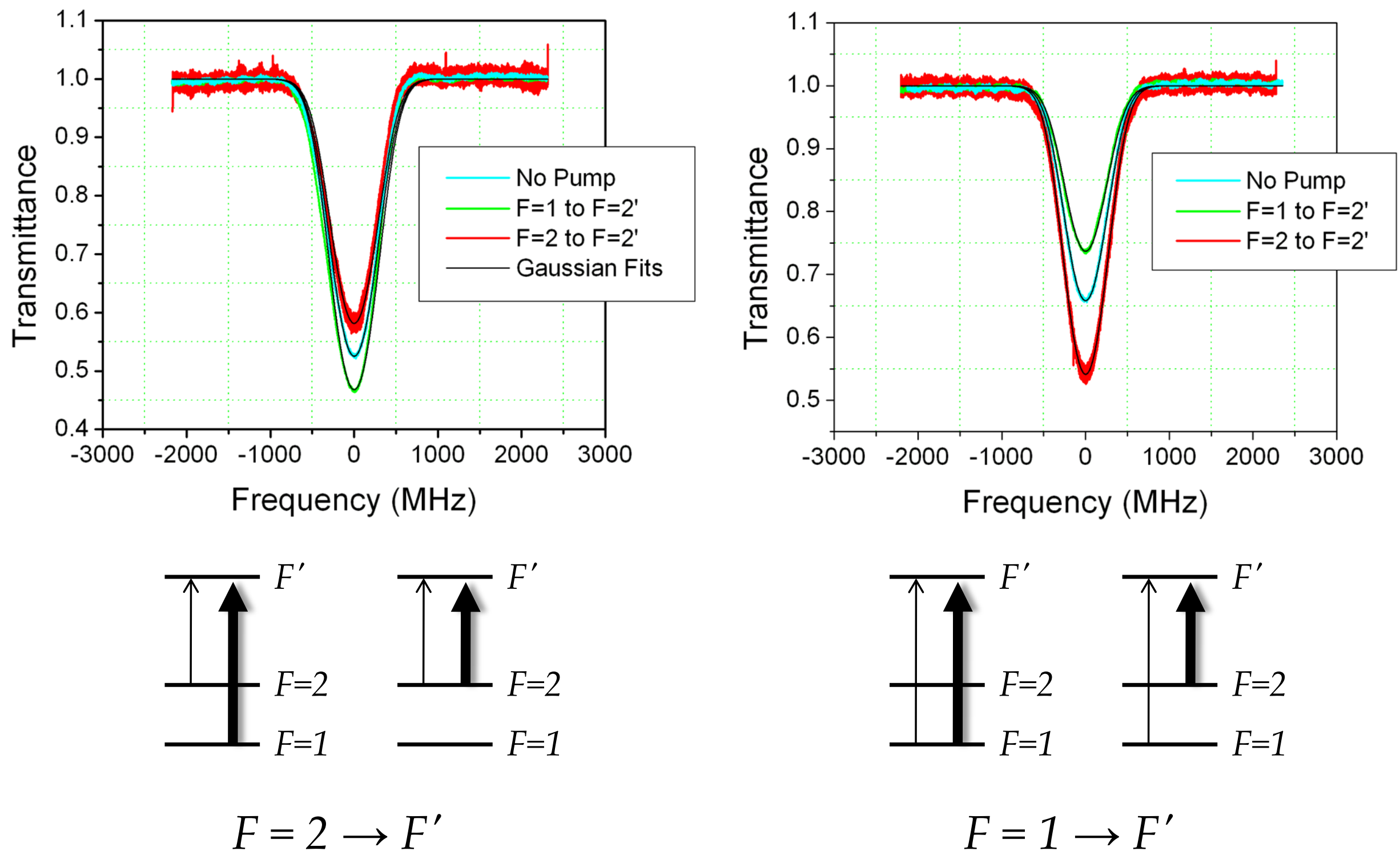

$F=2 \rightarrow F^{\prime}$

$F=1 \rightarrow F^{\prime}$ 


\section{Scale Factor: Tuning by Pumping}

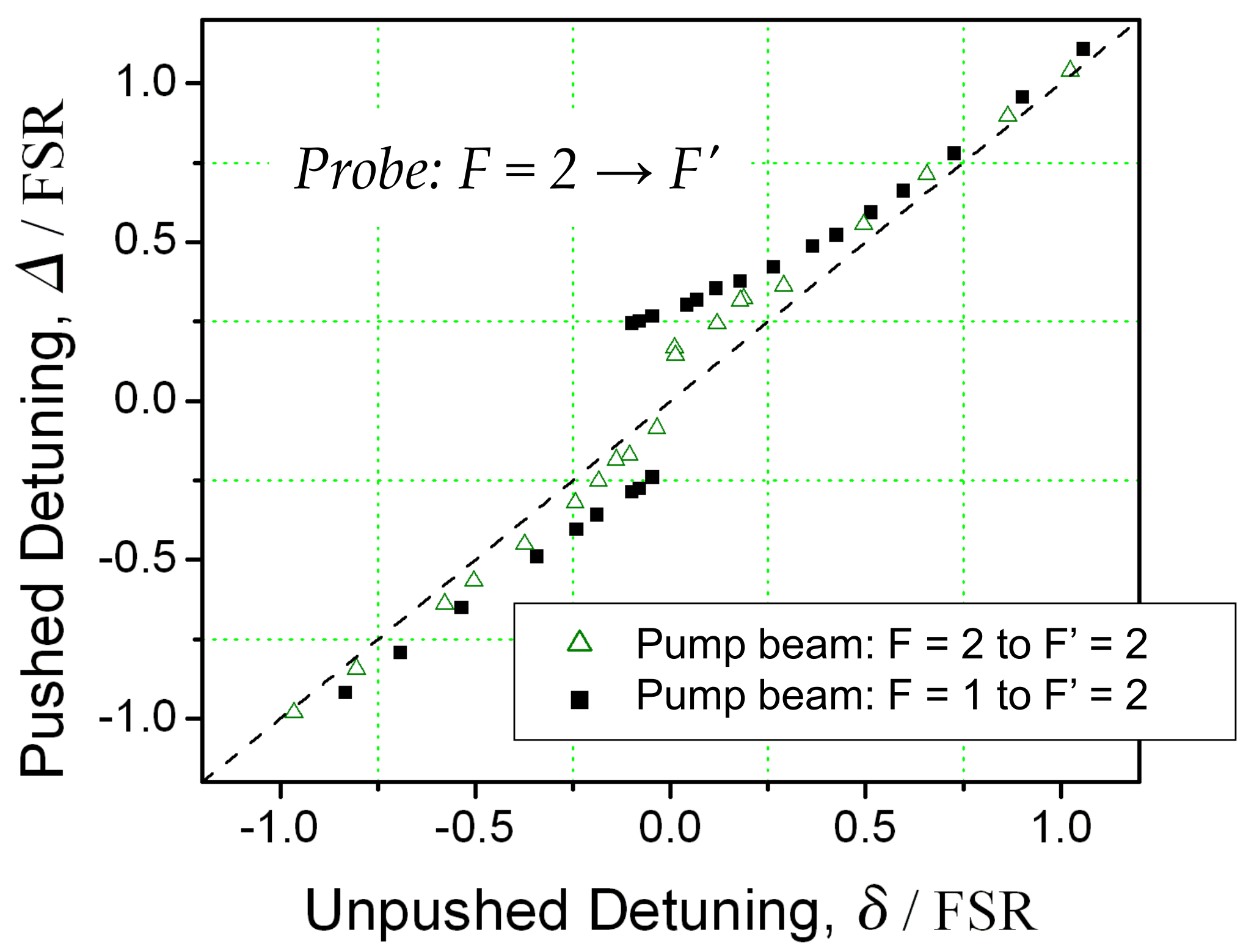




\section{Scale Factor: Tuning by Pumping}

$$
\text { Probe: } F=1 \rightarrow F^{\prime}
$$
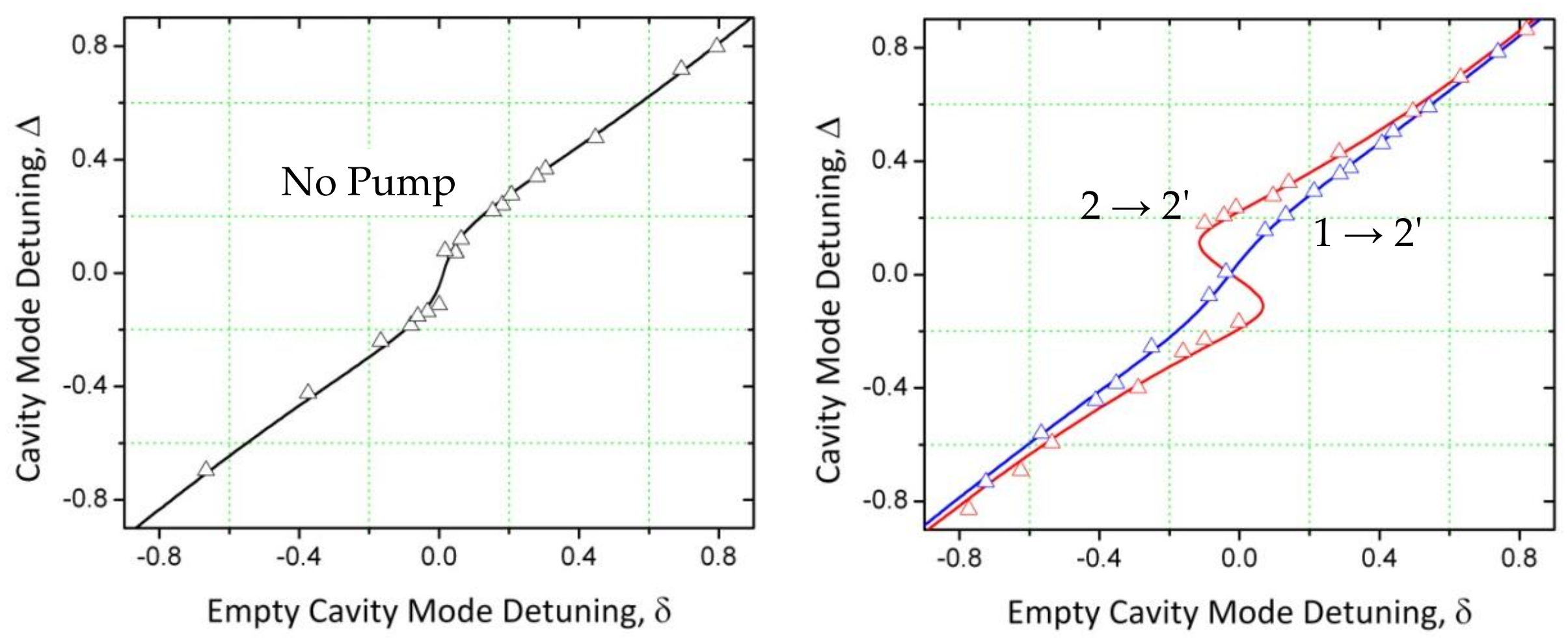

> Hyperfine repumping increases probe absorption and scale factor

$>$ Continuous tuning by varying pump intensity. 


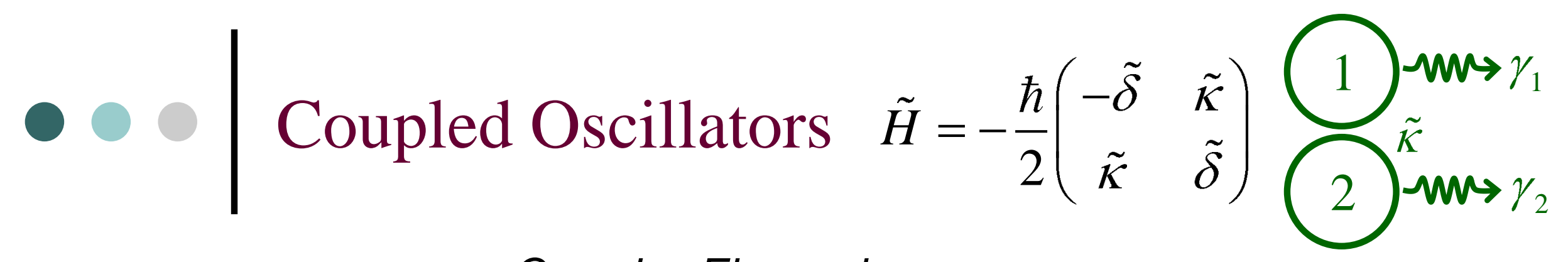

Complex Eigenvalues:

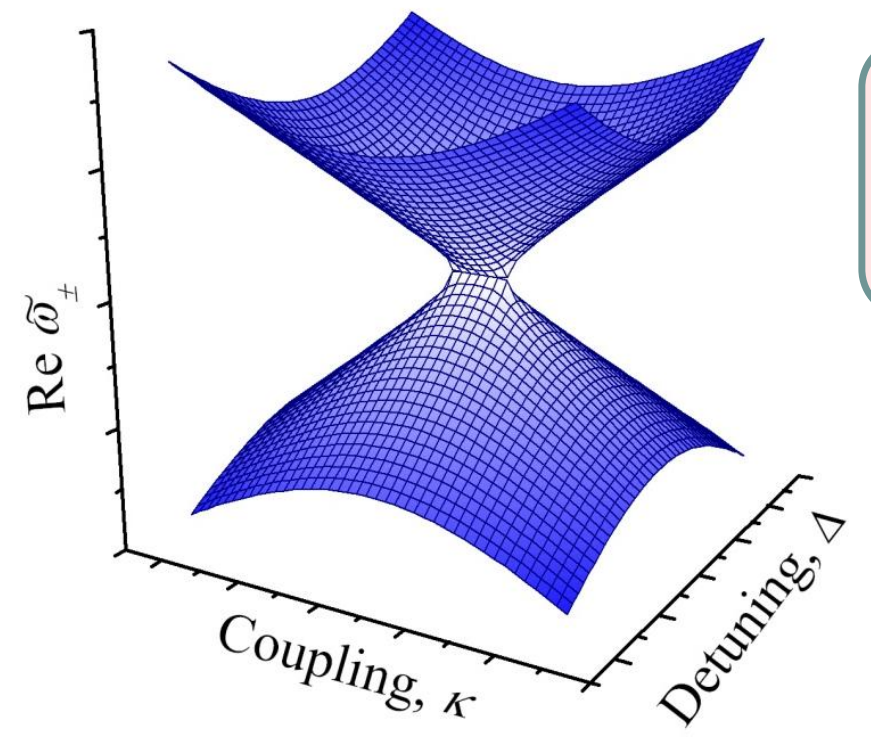

Super-exceptional coupling

$$
|\gamma / 2|<\kappa
$$

Frequency Anti-Crossing \& Width Crossing

$$
\begin{gathered}
\tilde{\omega}_{ \pm}=\tilde{\omega}_{\text {avg }} \pm \frac{\tilde{\Omega}}{2} \\
\tilde{\Omega}=\left[\tilde{\delta}^{2}+\tilde{\kappa}^{2}\right]^{1 / 2} \\
\tilde{\delta}=\delta-i \frac{\gamma}{2}
\end{gathered}
$$

Exceptional coupling

$$
|\gamma / 2|=\kappa
$$

Frequency \& Width Crossing

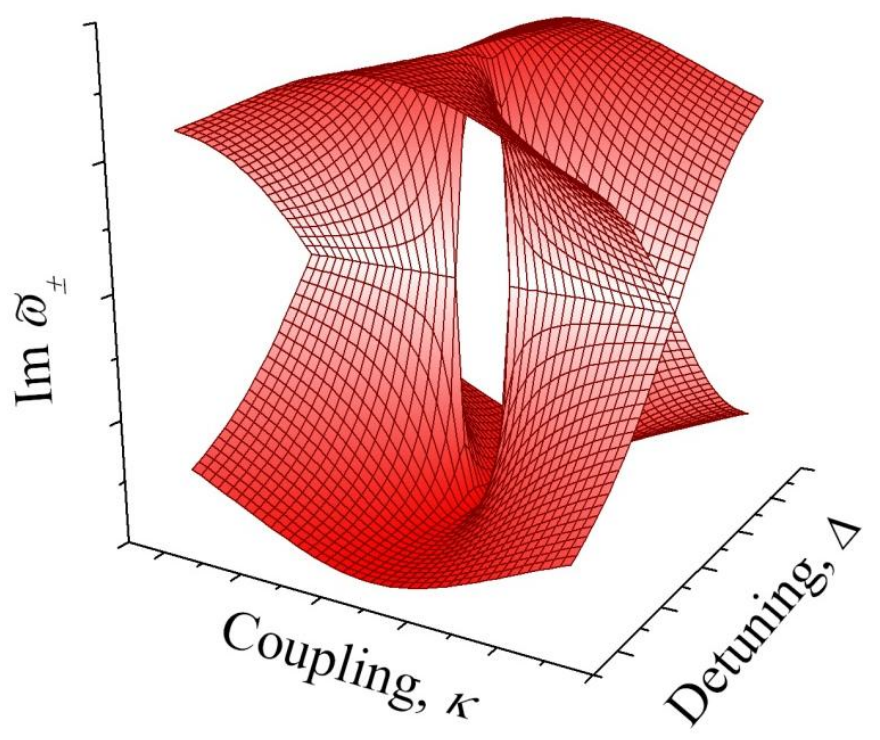

Sub-exceptional coupling

$$
|\gamma / 2|>\kappa
$$

Frequency Crossing \& Width Anti-Crossing 


\section{Scale Factor Enhancement for Generic} Coupled Oscillators

$$
S(\delta) \equiv \frac{d \Delta}{d \delta}=\frac{1}{2\left[1+(\tilde{\kappa} / \tilde{\delta})^{2}\right]^{1 / 2}}+c . c .
$$
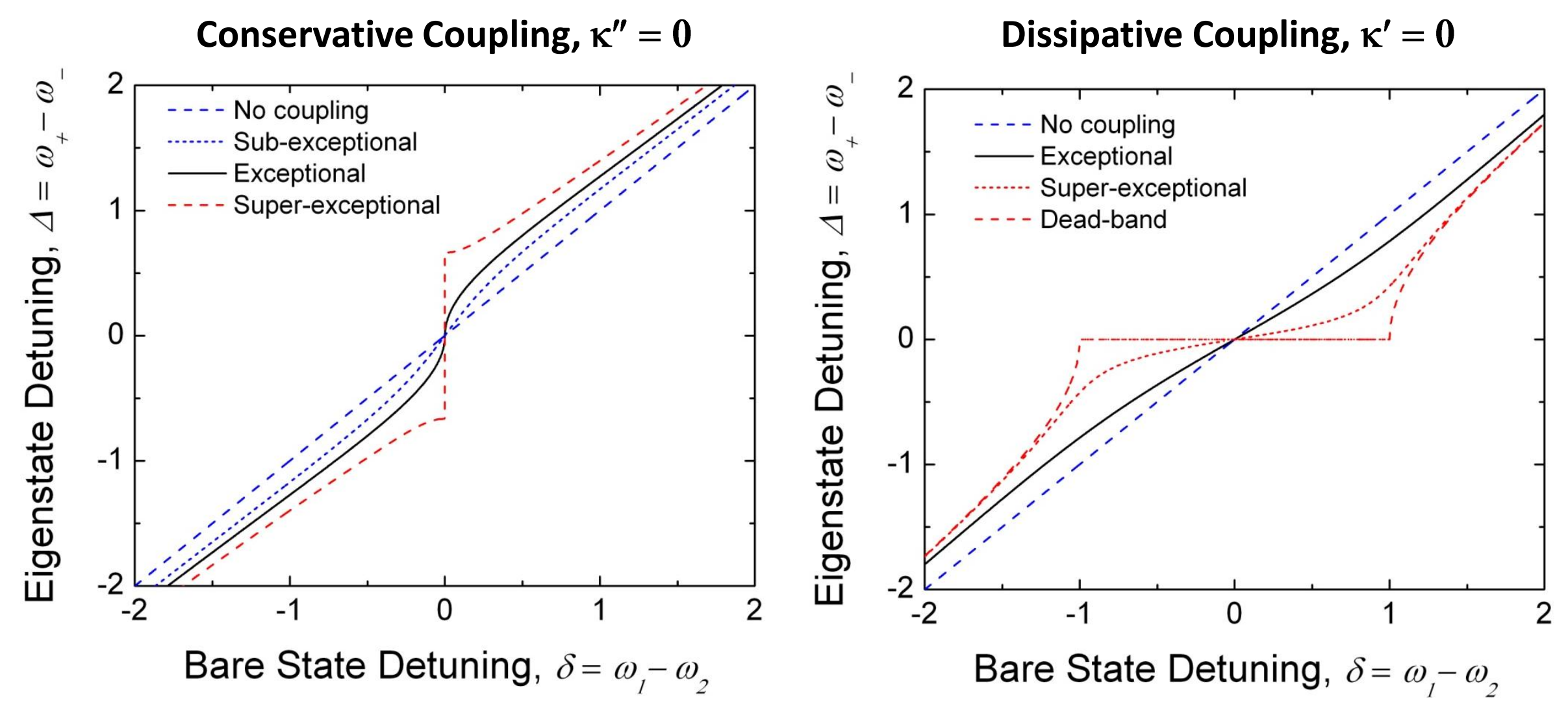

Exceptional point coupling $\rightarrow$ Scale factor pole 


\section{Decreasing the Scale Factor}
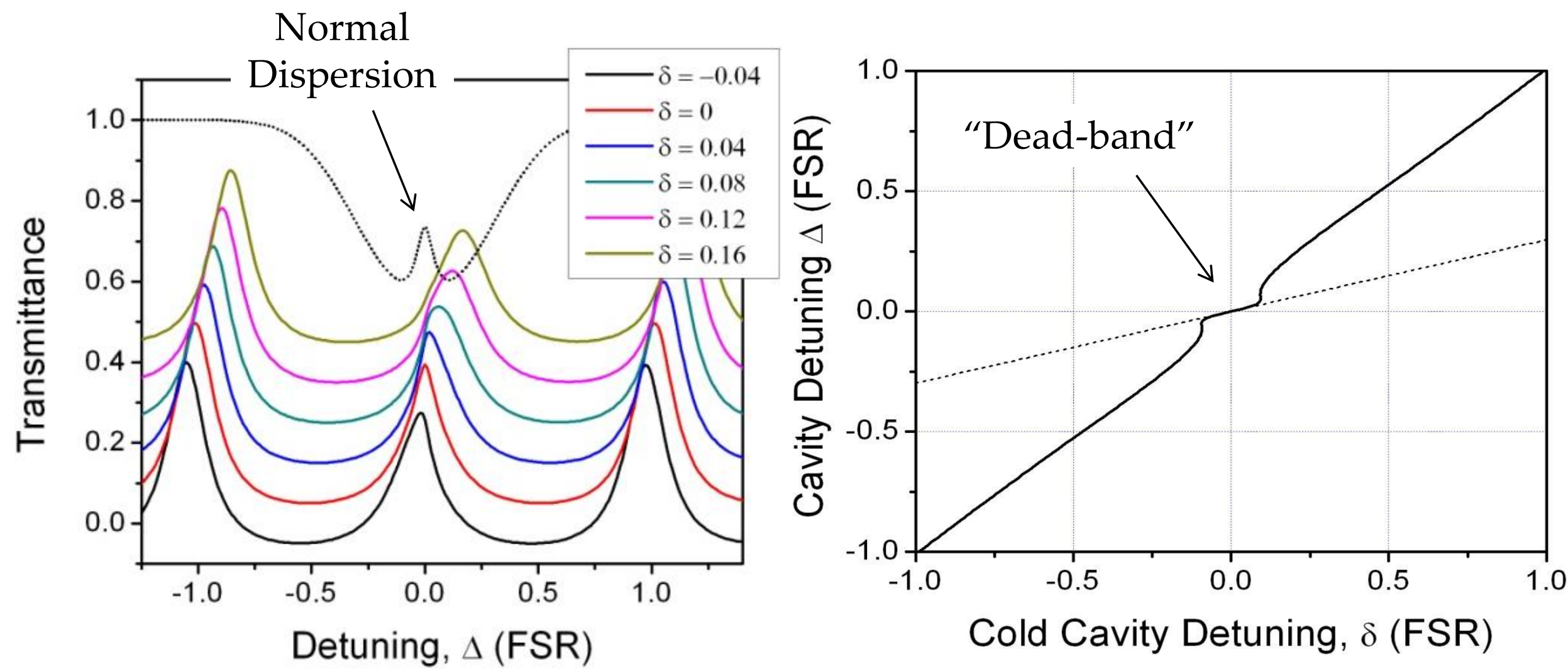

$>$ mode is pulled and trapped by normal dispersion, then abruptly released and pushed by broader anomalous dispersion feature.

$>$ Scale factor is significantly reduced near resonance. 


\section{Normal Dispersion by VSOP}
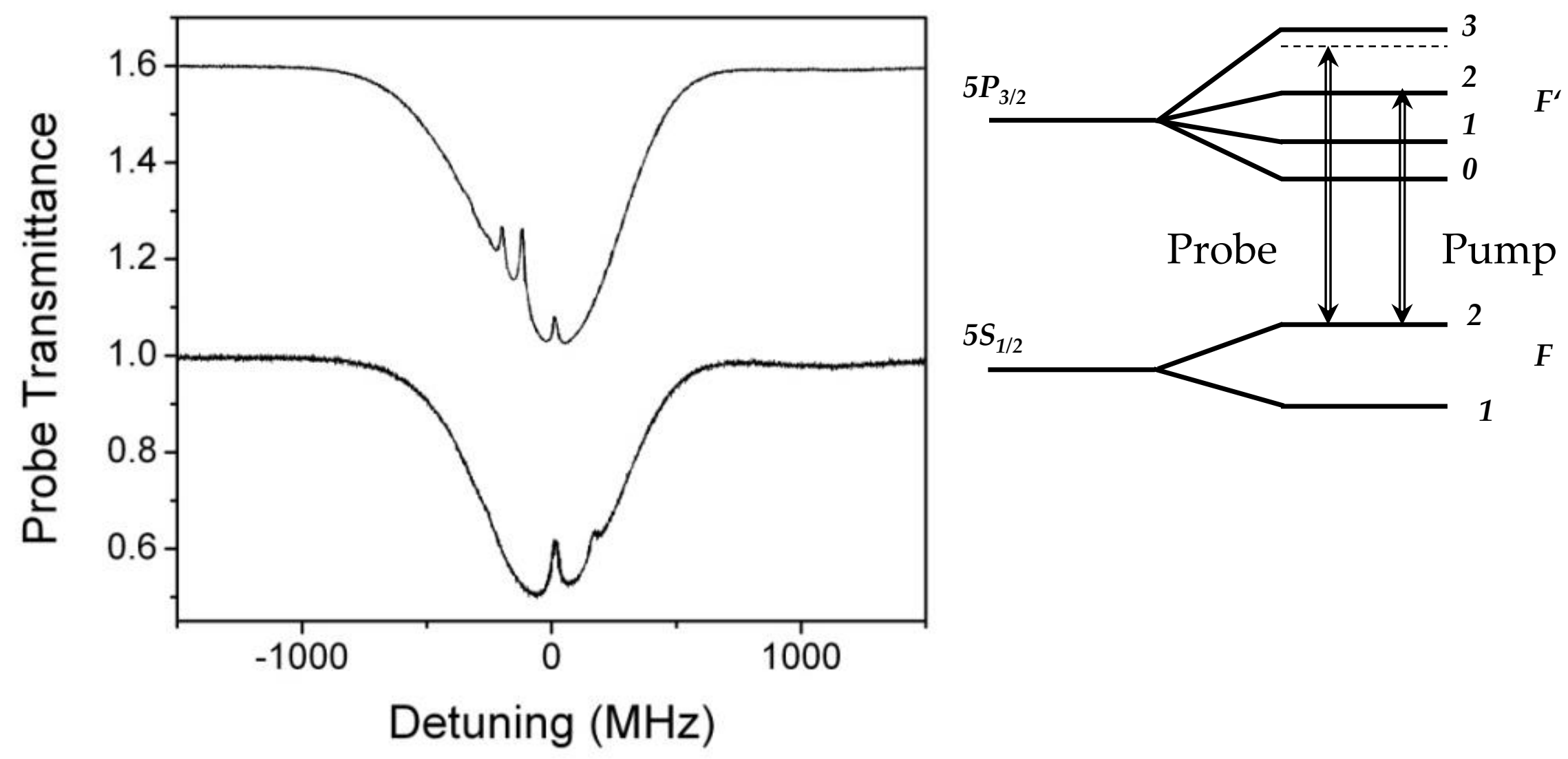

$>$ VSOP resonances are optical pumping holes of tunable width and depth, i.e., tunable dispersion. 


\section{Tuning by Coherent Control of Absorption}

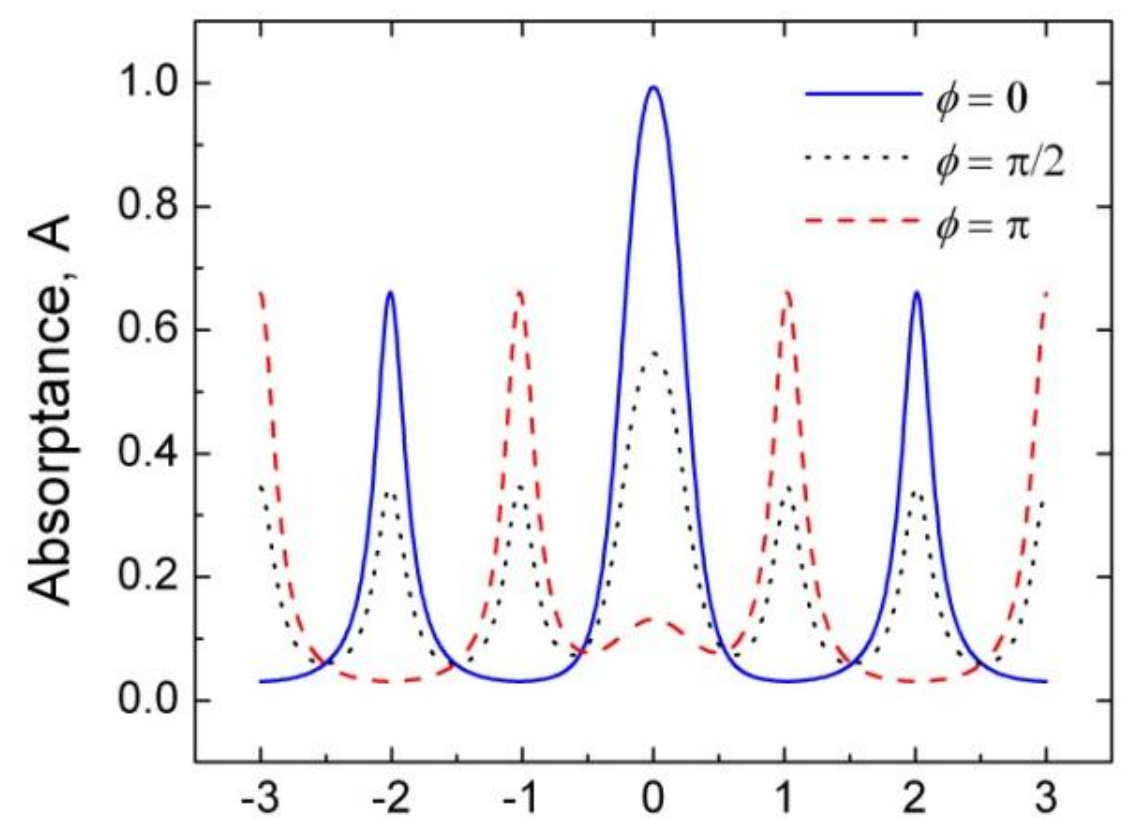

Cavity Mode Detuning, $\Delta$

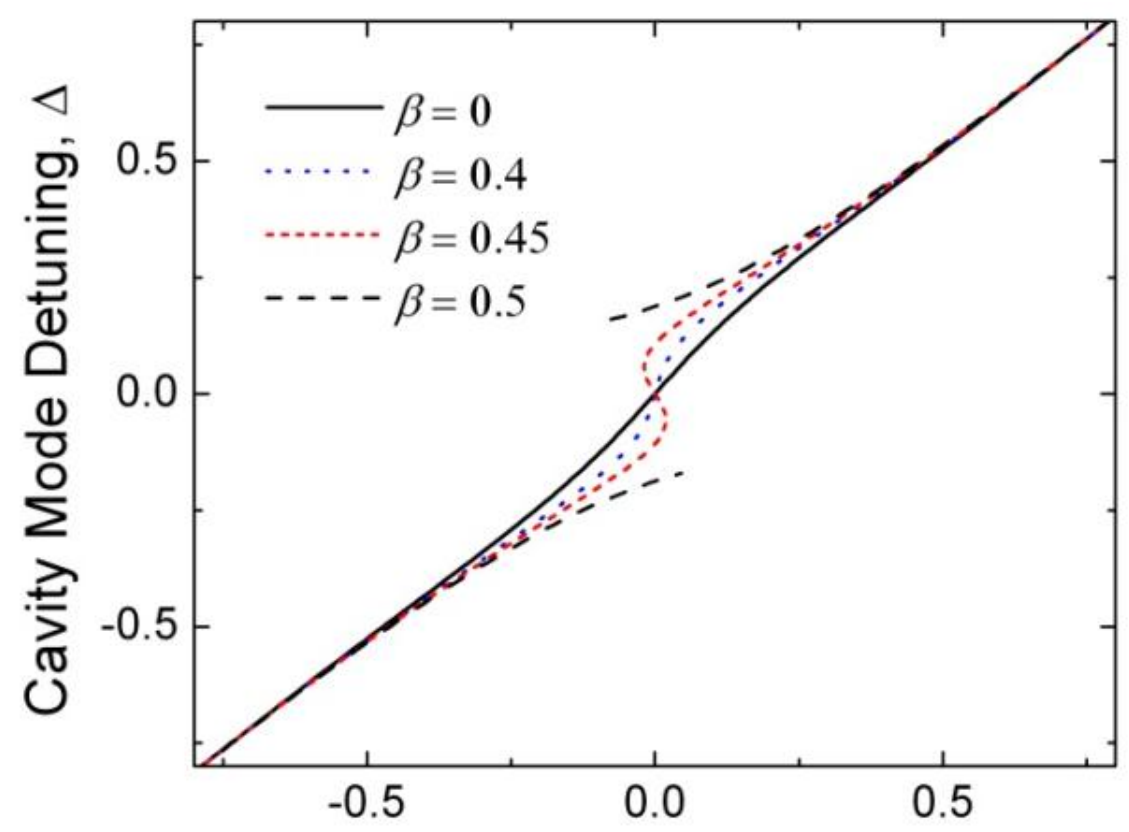

Empty Cavity Mode Detuning, $\delta$

$>A$ second beam incident on cavity increases cavity absorptance, which in turn increases gyro scale factor.

$>$ Linear effect, independent of choice of intracavity material, only moderate intensities required on second beam. 


\section{Closed Loop Experimental Setup}

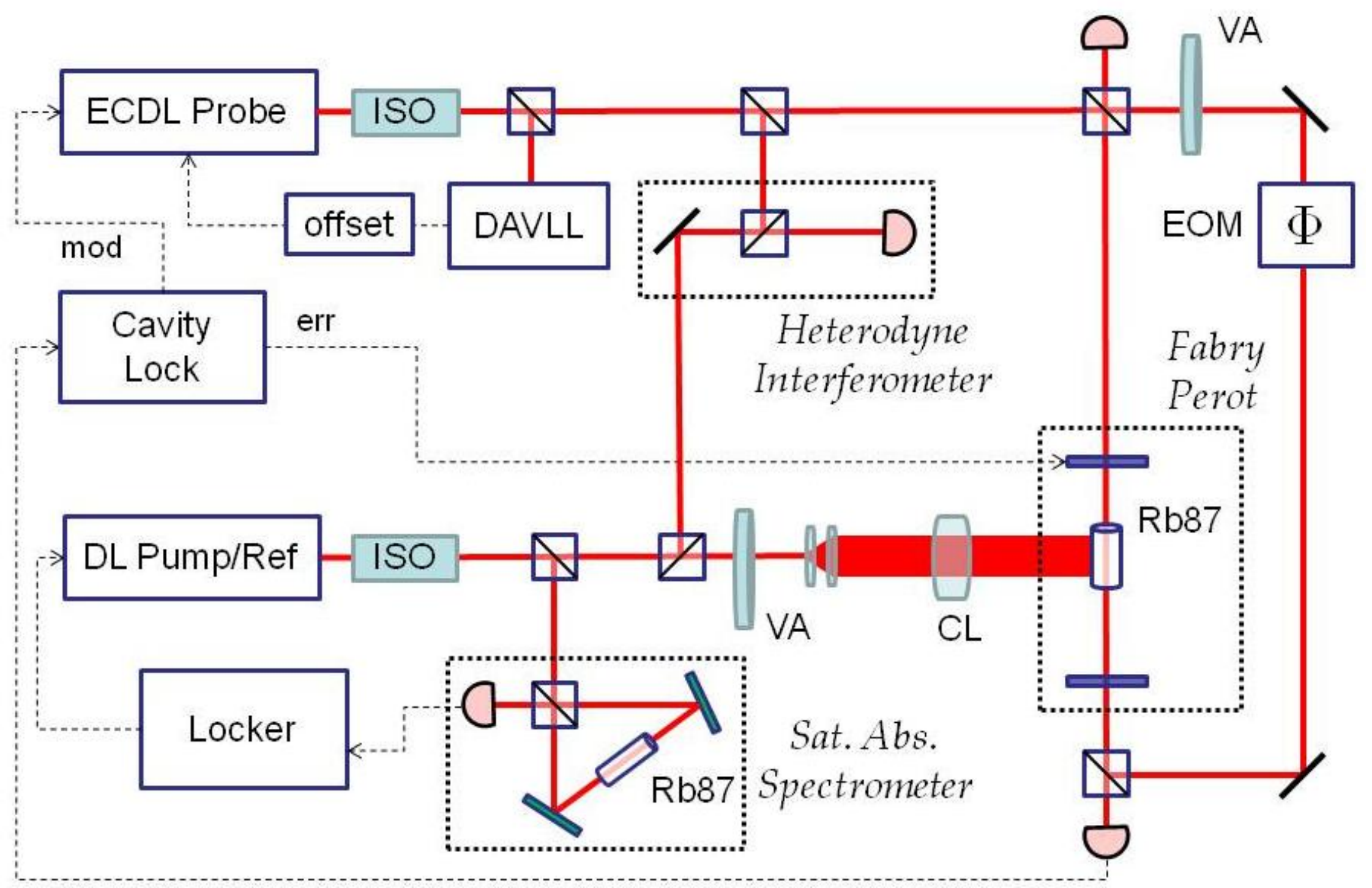




\section{Conclusions}

- The sensitivity of a passive cavity can be enhanced due to anomalous dispersion (even more so than in the case of a laser due to the absorption) and controlled by a repumping or interfering beam. But mode splitting occurs when atom-cavity coupling is too large.

- The sensitivity of any coupled oscillator system is enhanced near the exceptional point (scale factor pole, critical gain/anomalous dispersion) A dispersive medium in a cavity is a coupled oscillator.

- Velocity-selective optical pumping can be used to produce a normal dispersion feature that can reduce scale factor.

- The cavity can be locked to the laser, which in turn is locked to the atomic resonance, resulting in closed-loop operation. 


\section{Acknowledgements}

Collaborators: $>$ C. Schambeau (UAH)

$>$ A. Odutola (AAMU)

$>$ A. Toftul (UNL)

D K. Myneni, H. Chang (AMRDEC)

$>$ J.C. Diels (UNM)

Support:

D U.S. Army Independent Laboratory In-House Research (ILIR) Program

$>$ NASA Administrator's Fellowship Program

$>$ NASA Nebraska Space Grant Consortium

Optical Sciences

University of New Mexico
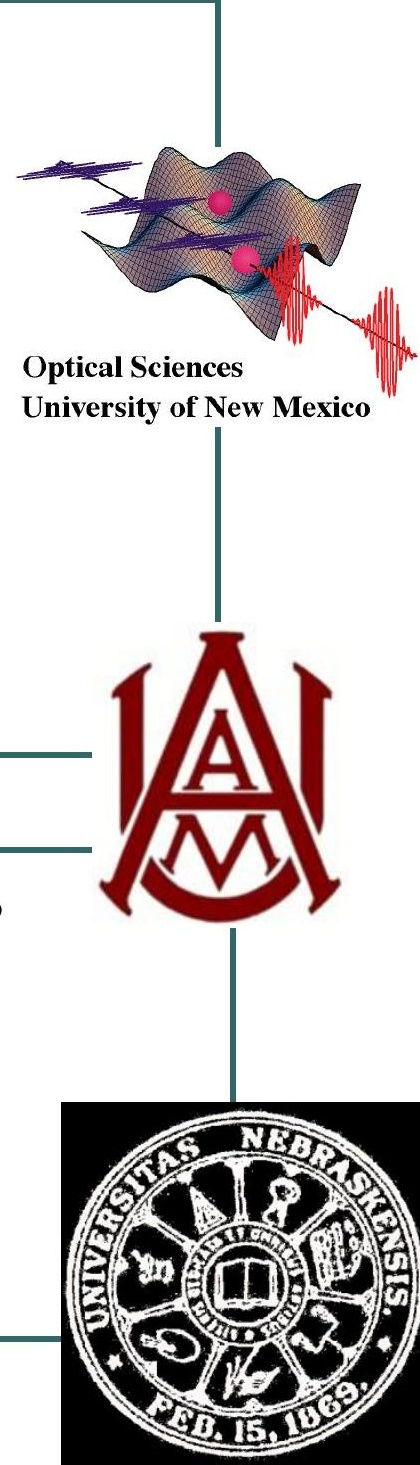